\title{
DPCrowd: Privacy-preserving and Communication-efficient Decentralized Statistical Estimation for Real-time Crowd-sourced Data
}

\author{
Xuebin Ren, Chia-Mu Yu, Wei Yu, Xinyu Yang, Jun Zhao, and Shusen Yang
}

\begin{abstract}
In Internet of Things (IoT) driven smart-world systems, real-time crowd-sourced databases from multiple distributed servers can be aggregated to extract dynamic statistics from a larger population, thus providing more reliable knowledge for our society. Particularly, multiple distributed servers in a decentralized network can realize real-time collaborative statistical estimation by disseminating statistics from their separate databases. Despite no raw data sharing, the real-time statistics could still expose the data privacy of crowd-sourcing participants. For mitigating the privacy concern, while traditional differential privacy (DP) mechanism can be simply implemented to perturb the statistics in each timestamp and independently for each dimension, this may suffer a great utility loss from the real-time and multi-dimensional crowd-sourced data. Also, the real-time broadcasting would bring significant overheads in the whole network. To tackle the issues, we propose a novel privacy-preserving and communication-efficient decentralized statistical estimation algorithm (DPCrowd), which only requires intermittently sharing the DP protected parameters with one-hop neighbors by exploiting the temporal correlations in real-time crowd-sourced data. Then, with further consideration of spatial correlations, we develop an enhanced algorithm, DPCrowd+, to deal with multidimensional infinite crowd-data streams. Extensive experiments on several datasets demonstrate that our proposed schemes DPCrowd and DPCrowd+ can significantly outperform existing schemes in providing accurate and consensus estimation with rigorous privacy protection and great communication efficiency.
\end{abstract}

Keywords-Differential privacy, decentralized statistical estimation, real time, communication efficiency, crowd-sourced data

\section{INTRODUCTION}

With the proliferation of smart devices and communication technologies, massive crowdsensing data can be acquired in real time, including industrial data in Industrial Internet-ofThings (IIoTs) [1], [2], proximity sensing data in Internetof-Vehicles (IoVs) [3], [4], and IoT-based health data [5], [6]. The aggregate statistics [7], [8] of these real-time data can provide valuable knowledge (e.g., popular business sites, disease outbreaks, and traffic dynamics [9], [10]) and facilitate intelligence for numerous smart-world systems, including smart industry or smart cities [11], [4]. Nonetheless, these data could be crowd-sourced and stored at peer organizations (e.g., companies and hospitals) or edge servers (e.g., smart vehicles) [12] as isolated data silos, which are difficult to be

X. Ren, X. Yang, S. Yang are with Xi'an Jiaotong University. (\{xuebinren, yxyphd, shusenyang\}@mail.xjtu.edu.cn)

C. Yu is with National Chiao Tung University. (chiamuyu@gmail.com).

W. Yu is with Towson University. (wyu@towson.edu)

J. Zhao is with Nanyang Technological University. (junzhao@ntu.edu.sg) thoroughly aggregated and fully utilized [13]. Therefore, it is essential to help multiple distributed parties to achieve realtime statistical analysis (or statistical parameter estimation) from their separately crowd-sourced databases.

Different from conventional statistical parameter estimation that relies on a central server to process all crowd-sourced data [14], [10], these peer servers often belong to no central entity but equal to each other, thus can only form a decentralized network with no mutual trust [15], [3], as shown in Fig. 1. Distributed or decentralized statistical parameter estimation [16], [17], [7], [8] has been studied in wireless sensor networks to infer the environment parameters by sharing intermediate statistics, which, however, may still expose the sensitive information. Particularly, for the distributed crowdsourcing servers, statistics sharing among multiple servers may disclose the sensitive information of crowd-source users or provide extra information for malicious or adversary compromised servers [1].

Differential privacy (DP), as the de-facto paradigm for privacy preservation with rigorous guarantee [18], [19], has received considerable attention in the privacy protection of monitoring or crowd-sourced data, focusing on either data publication [20], [21], [22], [23] or statistical aggregation [24], [9], [10], [25]. Nonetheless, most of the existing works are considered in the context of single-server application [24], [9], or rely on a central coordinator [10], [20], or only achieve one-time data publication [20], [22], [23], or conduct multiple rounds of computation while suffering from severe privacy degradation [26], [27], [28]. None of them can be directly adopted for our application scenario, in which fully decentralized servers conduct real-time statistical estimation without any central entity. Thus, this motivates us to design a novel differential private and communication efficient framework of real-time statistical estimation from crowd-sourced data stored at multiple distributed servers in a fully decentralized network.

Design Challenges. The main challenges in developing such a framework with DP can be summarized as follows.

- Huge communication cost. To achieve consensus estimation for distributed servers in a decentralized network, a straightforward method is to let each server release its own aggregate statistics to all other servers hop by hop at each timestamp. Nonetheless, besides privacy concerns, continuous multi-hop broadcast incurs both tremendous communication overhead and high delay.

- Real-time data release. The global information often needs to be derived in a real-time fashion (e.g., the 
traffic condition or epidemic disease outbreak). Nonetheless, according to the sequential composition theorem of DP [29], naive DP protection on continuous data stream causes severe utility loss or extravagant privacy budget consumption [30].

- Multi-dimensional Data. The aggregate statistics may be multi-dimensional, reflecting different aspects of the environment. Nonetheless, with the increase of data dimensions, the data stream would be sparse and lead to both high computational complexity and low data utility for many existing privacy-preserving algorithms [9].

Contributions. Our contributions are summarized below.

- We propose DPCrowd, an efficient framework of realtime differentially private decentralized statistical estimation for multiple distributed servers with separately crowd-sourced datasets. To the best of our knowledge, this is the first work realizing real-time decentralized statistical estimation with both privacy protection and communication efficiency.

- We leverage the Laplace mechanism and Kalmanconsensus information filter to realized privacy protection and communication reduction for real-time decentralized statistical estimation with fast convergence and consensus estimation. By further adopting adaptive sampling based intermittent communication strategy, DPCrowd can achieve statistical estimation with much higher utility privacy tradeoff and lower communication cost.

- Based on DPCrowd, we further present DPCrowd+ to deal with multi-dimensional infinite data streams. DPCrowd+ satisfies $w$-event DP for infinite streams and mitigates the sparsity issue in multi-dimensional data, thus further enhancing the utility for statistical estimation on multi-dimensional streams.

- We conduct extensive experiments on both synthetic and real-world datasets. The experimental results demonstrate that DPCrowd and DPCrowd+ can not only achieve superior estimation accuracy under the given privacy guarantees, but also offer desirable estimation consensus with low communication cost.

The remainder of this paper is organized as follows: In Section II, we conduct a brief literature review of related works. In Section III, we introduce models and formalize the problem. In Section IV, we provide some preliminaries. In Section V, we introduce our baseline and enhanced schemes. In Section VI, we conduct the privacy, utility, communication latency and cost analysis of our schemes. In Section VII, we describe the performance evaluation results. Finally, we conclude the paper in Section VIII.

\section{RELATED WORK}

In the following, we review some works that are relevant to our study.

DP for Data Stream Publication. Dwork et al. initiated the theoretical study of DP [31] on streaming data release (or publication) [32], [30]. They proposed two DP notions, namely event-level and user-level DP. The former hides a single event and the latter hides all the events of any user.
Mir et al. [33] studied estimating distinct counts, moments, and heavy hitters, which is also studied by Chan et al. [34]. In addition, Fan et al. [24] presented FAST to achieve DP aggregate monitoring in the sampling-and-filtering framework. Chen et al. [35] presented PeGaSus to achieve event-level DP in the framework of perturb-group-smooth. Likewise, Kellaris et al. [36] addressed the shortcoming of event-level DP and user-level DP, and proposed a new notion of $w$-event DP, which can be thought of as a sliding window version of DP on the infinite data stream. Wang et al. [9] proposed RescueDP by applying the idea of $w$-event DP to FAST. Beyond that, the authors further enhanced RescueDP with advanced techniques, such as recurrent neural network in time-series analysis and dynamic programming for dynamic grouping, which demonstrate much better performance [37]. All these studies are considered in the context of a singleserver application.

DP for Distributed Data Publication. Most above data publication studies focus on streaming data in a centralized setting and are not practical for the distributed scenarios. Early studies attempt to achieving DP via adding partial noise at distributed servers [38]. For example, Goryczka et al. [39] conducted a comparative study on secure data aggregation with DP in a distributed setting. Alhadidi et al. [40] proposed to privately publish horizontally partitioned data with integration of DP and secure multi-party computation. Hong et. al. [41] proposed collaborative generation algorithms for search logs at different parties with $(\varepsilon, \delta)$-DP. Su et al. [20] presented a DP solution to publishing high-dimensional, but vertically split data in a distributed setting. Nonetheless, these schemes mainly deal with static data. Further, Wang et al. [10] rebuilt RescueDP [9][37] and proposed a distributed framework of DADP by introducing multiple agents between the crowdsourcing users and the central server. Nonetheless, it still relies on the coordination of a central server and is not fully decentralized. Beyond the above studies, local differential privacy (LDP) has also been a promising paradigm for largescale crowd-sourcing systems for various applications [42], [22].

DP for Distributed Parameter Estimation. There have been a few studies on private distributed or decentralized parameter estimation recently. For example, Belmega et al. [43] explored an information-theoretic approach to obtain the state estimation between two parties with privacy. Huang et al. [26], [27] proposed a class of iterative algorithms for solving the private distributed optimization problem. Recently, a variety of privacy-preserving distributed (collaborative) learning or federated learning (FL) [44], [45], [46], [47], [48] approaches have emerged as new solutions to privately learn from distributed datasets. For example, Geyer et al. [46] proposed to achieve client level DP for distributed FL clients by injecting noise to the aggregated update models of distributed clients, where moment accountant mechanism is also used for tightly tracking the privacy loss. Truex et al. [44] combined both techniques of DP and secure multiparty computation to reduce the noise growth while maintaining effective privacy guarantee. Likewise, Zhao et al. [45] achieved privacy-preserving distributed collaborative deep learning via not only running 
privacy-preserving stochastic descent gradient independently on distributed datasets using object perturbation on loss function, but also privately selecting reliable participants via the exponential mechanism. These methods can allow massive distributed data utilization with privacy preservation, which, however, are mainly considered in a batch learning scenario instead of streaming setting. To address this issue, Li et al. [28] presented a distributed online learning framework with DP. Nonetheless, temporal correlations in the dynamic estimation were hardly considered in these studies.

Unlike the above studies, we aim to design a privacypreserving and communication efficient framework of realtime decentralized statistical estimation for multiple distributed servers with crowd-sourced data streams, which can be widely used in IoT-driven smart-world systems. There is a controversy [49], [50], [51] over what does DP guarantee for correlated data streams due to different understanding of privacy definition. Some recent works [50], [52] suggested that DP offers a weaker bound on privacy loss when data records are correlated. Nonetheless, similar to [35], in this paper, we emphasize to design privacy-preserving mechanisms based on general DP definitions [30] with privacy parameter $\varepsilon$ while minimizing the statistical estimation error.

\section{Models AND PRoblem Definition}

In this section, we first introduce system model, communication model, data model, data model, adversary model and then present the problem definition.

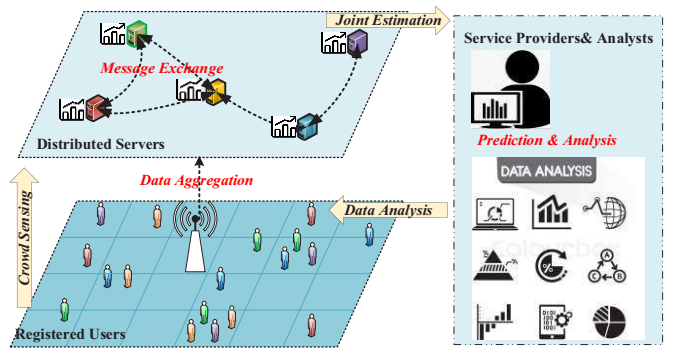

Fig. 1: Decentralized Statistical Estimation from Crowdsourced Data

System Model. As shown in Fig. 1, we consider there are $m$ distributed servers that provide geo-location services to a population of $n$ crowd-sourcing users $\{1, \ldots, n\}$ scattered in an area, which is divided into $d$ disjoint regions. Each time $t$, each user randomly registers at one of the distributed servers, and uploads the check-in information with the secure connection technology. Each server $\mathrm{SP}_{i}(i=1, \ldots, m)$ then collects the crowd-sourced data from its corresponding user group $G_{i}(t) \subseteq\{1, \ldots, n\}$ with the population of $\left|G_{i}(t)\right|$. Assume that all the users generally follow the same mobility model (i.e., the same transition probability from one region to another) when the regions are coarsely divided. In this paper, these servers are considered to be connected in a decentralized network and interested in the real-time population distribution among regions.
Communication Model. Though mostly static, we assume a general scenario, in which the communication network among distributed servers is dynamic and evolves with time (e.g., vehicular networks). We assume all servers communicate with each other based on a $m \times m$ time-variant adjacent matrix $\mathcal{E}(t)$. We abstract the communication graph for $i^{t h}$ server at timestamp $t$ as

$$
\mathcal{G}_{i}(t)=\left\{(i, j): e_{i j}(t) \in \mathcal{E}(t)\right\} .
$$

Here, the element $e_{i j}(t)=1$ means that there exists a communication between server $i$ and $j$ at the timestamp $t$ while $e_{i j}(t)=0$ means that no communication between them. We assume that at every timestamp, the graph has no isolated server; i.e., for each $i=1, \ldots, m$, there exists $j \neq i$ such that $e_{i j}(t)=e_{j i}(t)=1$.

Data Model. Let $D_{t}$ be a two-dimensional matrix with the size of $n \times d$ at timestamp $t$. Denote $D_{i, t}=\left[D_{i, t}\left[u_{1}^{i}\right]^{T} \ldots D_{i, t}\left[u_{\left|G_{i}(t)\right|}^{i}\right]^{T}\right]^{T}$ as the two-dimensional database at the $i^{t h}$ server $\mathrm{SP}_{i}$ at time $t$, with the size of $\left|G_{i}(t)\right| \times d$, where $D_{i, t}\left[u_{j}^{i}\right]\left(1 \leq j \leq\left|G_{i}(t)\right|\right)$ denotes the $j^{\text {th }}$ row of $D_{i, t}$, which corresponds to $\mathrm{SP}_{i}$ 's registered users $u_{j}^{i} \in G_{i}(t)$. In $D_{i, t}$, each row corresponds to a registered user in $G_{i}(t)$ and each column corresponds to a region. The value of $D_{i, t}(p, q)$ is 1 refers to the case that the $p^{t h}$ user in $G_{i}(t)$ appears at the $q^{t h}$ region at time $t$, and 0 otherwise. Since any user can appear at exactly one region at the same time, each row in $D_{i, t}$ also contains at most one 1 .

Adversary Model. We focus on the data privacy of crowdsourcing users in decentralized statistical estimation. We assume the crowd-sourcing users trust on the distributed servers, at which they registered. This assumption is common as the users may have to contribute their data to the servers for certain personalized services, i.e., online recommendation or real-time navigation. Nonetheless, they wish better service quality while minimizing their privacy risks. Thus, each user would consider his/her unregistered servers or any third-party analysts are potential honest-but-curious adversaries, which honestly follow the mechanism, but try to infer the private information from his/her register server. This adversary model is practical in a decentralized network where distributed servers belong to different individuals or organizations, which do not have mutual trust.

Problem Definition. Let $\mathbf{r}(t)=f\left(D_{t}\right)=$ $\left(r^{1}(t), r^{2}(t), \ldots, r^{d}(t)\right)$ denote the true statistics (e.g., $r^{k}(t)$ denote the total number of users) over $D_{t}$ at time $t$ in the $k^{t h}$ region $(k=1,2, \ldots, d)$, where $f$ is an aggregate function (e.g., sum) applied to all $d$ dimensions. However, $\mathbf{r}(t)$ cannot be accurately obtained by any distributed server with consensus, which only has partial knowledge of all crowdsourcing users. In particular, the $i^{t h}$ server can only aggregate its registered users' data to produce its own aggregate statistics $\mathbf{x}_{i}(t)=f\left(D_{i, t}\right)$ and may directly estimate $\mathbf{r}(t)$ from $\mathbf{x}_{i}(t)$. Nonetheless, due to partial samples and no coordination, the estimation would be rather rough and vary extravagantly among the distributed servers. Thus, collaborative estimation from multiple distributed servers seems to be promising. Nonetheless, severe privacy risks under the above adversary model and communication overheads in a time-varying 
decentralized network may still prevent the collaborations among servers. To further encourage active collaboration, the distributed servers can also be rewarded with incentives mechanisms based on their contributions recorded by some distributed ledgers (such as Blockchain) [53]. However, this is beyond our focus of privacy preservation in this paper.

Therefore, based on aforementioned system models and assumptions, our problem can be formalized as: with the partial stream datasets $D_{1, t}, D_{2, t}, \ldots, D_{m, t}$ at $m$ distributed servers in a time-varying decentralized network $\mathcal{G}_{i}(t)$, we focus on helping the mutually untrusted distributed servers to communication-efficiently estimate the accurate overall statistics $\mathbf{r}(t)$ in real-time with consensus while guaranteeing differential privacy for crowd-sourcing users registered at each distributed server.

\section{PRELIMINARIES}

In this section, we provide some background about the notion of differential privacy (DP), DP on data streams, as well as Kalman-Consensus information filter.

\section{A. Differential Privacy and Laplace Mechanism}

Differential privacy (DP) is a de-facto standard for data privacy. The rationale behind DP is that adding or removing any single data record will not have much influence on query results on the dataset. A formal definition of DP [18] is given below.

Definition 1 E-DP [18]: A randomized mechanism $\mathcal{M}$ satisfies $\varepsilon-D P$ if for any two neighboring datasets $D$ and $D^{\prime}$ that differ at most one data record, and for any possible outputs $O \subseteq \operatorname{Range}(\mathcal{M})$

$$
\operatorname{Pr}[\mathcal{M}(D) \in O] \leq e^{\varepsilon} \cdot \operatorname{Pr}\left[\mathcal{M}\left(D^{\prime}\right) \in O\right],
$$

where the probability is taken over $\mathcal{M}^{\prime}$ s randomness. Privacy budget $\varepsilon$ is a parameter for the tradeoff between privacy and utility. From Eq. (2), we see that smaller $\varepsilon$ means better privacy but lower utility.

Definition 2 Sensitivity [18]: For any function $f: \mathcal{D} \rightarrow \mathcal{R}^{d}$, the sensitivity of $f$ w.r.t $\mathcal{D}$ is defined as

$$
\Delta f=\max _{D, D^{\prime} \in \mathcal{D}}\left\|f(D)-f\left(D^{\prime}\right)\right\|
$$

for all $D$ and $D^{\prime}$ that differs on at most one record.

Laplace mechanism is the most popular scheme for DP, which adds carefully calibrated noise to query results [18]. In particular, the noise $v$ follows a zero-mean Laplace distribution $\mathcal{L}(b)$ with scale parameter $b$, which has the probability density function

$$
P(v \mid b)=\frac{1}{2 b} \exp \left(-\frac{|v|}{b}\right)
$$

Theorem 1 (Laplace Mechanism [18]) For any function $f$ : $\mathcal{D} \rightarrow \mathcal{R}^{d}$ on any dataset $D \in \mathcal{D}$, the Laplace Mechanism $\mathcal{M}$ that adds Laplace noise $\left\langle v_{1}, \ldots, v_{d}\right\rangle$ to the function output, i.e.,

$$
\mathcal{M}(D)=f(D)+\left\langle v_{1}, \ldots, v_{d}\right\rangle
$$

satisfies $\varepsilon-D P$, where $v_{k}$ for $k=1, \ldots, d$ is drawn from Laplace distribution $\mathcal{L}(\Delta f / \varepsilon)$ with $\Delta f$ as the sensitivity of $f(\cdot)$ and $\varepsilon$ as the privacy budget.

DP enjoys the following two useful properties [18].

Theorem 2 (Sequential Composition [18]). Let $\mathcal{M}_{1}, \ldots, \mathcal{M}_{T}$ be $T$ randomized mechanisms, each of which satisfies $\varepsilon_{t^{-}}$ DP. A sequence of mechanisms $\mathcal{M}_{t}$ over a database $D$ will guarantee $\sum \varepsilon_{t}-D P$.

Theorem 3 (Post-Processing [18]) Let $\mathcal{M}$ be a randomized mechanism satisfying $\varepsilon-D P$ and $f$ be an arbitrary function. Then, $f(\mathcal{M}(D))$ will still guarantee $\varepsilon-D P$.

\section{B. DP on Data Streams}

The most straightforward DP notion for data streams is event-level DP for infinite streams [32], [30], which aims to protect the presence of a particular event at the time $i$ in a stream with unlimited length. Another is user-level DP for finite streams, guaranteeing that the presence of any user is indistinguishable in an entire data stream during certain period. Event-level DP is weaker than user-level DP as it does not consider the correlation among events in consecutive timestamps. Nonetheless, user-level DP for finite streams may restrict many interruptible real-time applications that generate infinite streams, whereas event-level DP is sometimes insufficient. Thus, $w$-event privacy [36], an approximate user-level in a sliding window of $w$ continuous timestamps, is proposed as an alternative DP definition for data streams. In this paper, we try to cover both user-level DP for finite streams and $w$-event privacy for infinite streams.

Before giving the definition of $w$-event DP, we first introduce the definition of $w$-neighboring, which describes two streams differs in a window of $w$ timestamps. For an infinite data stream $S=\left[D_{1}, D_{2}, \ldots\right]$, we define its stream prefix at timestamp $t$ as $S_{t}=\left[D_{1}, D_{2}, \ldots, D_{t}\right]$.

Definition 3 (w-neighboring). For any positive integer $w$, two stream prefixes $S_{t}, S_{t}^{\prime}$ are defined as w-neighboring, if

1) for each $S_{t}[i], S_{t}^{\prime}[i]$ such that $i \in[t]$ and $S_{t}[i] \neq S_{t}^{\prime}[i]$, it holds that $S_{t}[i], S_{t}^{\prime}[i]$ are neighboring;

2) for each $S_{t}\left[i_{1}\right], S_{t}\left[i_{2}\right], S_{t}^{\prime}\left[i_{1}\right], S_{t}^{\prime}\left[i_{2}\right]$ with $i_{1}<i_{2}$, $S_{t}\left[i_{1}\right] \neq S_{t}^{\prime}\left[i_{1}\right]$ and $S_{t}\left[i_{2}\right] \neq S_{t}^{\prime}\left[i_{2}\right]$, it holds that $i_{2}-i_{1}+1 \leq w$.

Definition 4 (w-event $\varepsilon-D P)$. A mechanism $\mathcal{M}$ is w-event $\varepsilon$ $D P$, if for the given integer $w$, all output sets $O \subseteq \operatorname{Range}(\mathcal{M})$ and all w-neighboring stream prefixes $S_{t}, S_{t}^{\prime}$ with all $t$, it satisfies that

$$
\operatorname{Pr}\left[\mathcal{M}\left(S_{t}\right) \in O\right] \leq e^{\varepsilon} \cdot \operatorname{Pr}\left[\mathcal{M}\left(S_{t}^{\prime}\right) \in O\right] .
$$

\section{Kalman-Consensus Information Filter}

Kalman filter is an effective algorithm for estimating dynamic processes that contain statistical noise. In particular, an 
underlying dynamic process with noise can be formulated by a linear time-varying model (aka. process model)

$$
r(t+1)=A(t) \cdot r(t)+\omega(t),
$$

where $r(t)$ is the process state at time $t(r(0)$ is an initial state with a normal distribution $\left.N\left(\bar{r}(0), P_{0}\right)\right), \omega(t)$ is the noise sampled from a normal distribution $N\left(0, Q_{t}\right)$, and $A(t)$ is the transition matrix that describes the transitions of the process.

In a distributed network, each node $i$ can have an observation $x_{i}$ of the dynamic process with the following linear sensing model (aka. measurement model)

$$
x_{i}(t)=H_{i}(t) \cdot r(t)+v_{i}(t),
$$

where $H_{i}(t)$ is the observation matrix and $v_{i}(t)$ is the measurement noise assumed to follow a normal distribution $N\left(0, R_{t}\right)$.

The Kalman filter can be used for each node to estimate the true $r(t)$ independently. We denote $\hat{x}_{i}(t)$ and $\bar{x}_{i}(t)$ as estimate and prior estimate of $r(t)$, respectively, for node $i$. Then, the estimate $\hat{x}_{i}(t)$ of $r(t)$ can be given as a linear combination of the prior estimate $\bar{x}_{i}(t)$ and the measurement $x_{i}(t)$

$$
\hat{x}_{i}(t)=\bar{x}_{i}(t)+K_{i}(t)\left(x_{i}(t)-H_{i}(t) \bar{x}_{i}(t)\right),
$$

where $K_{i}(t)$ is called Kalman gain and adjusted to minimize the posterior error covariance at each timestamp. Particularly, the prior estimate $\bar{x}_{i}(t)$ can be predicted according to the process model (Eq. (7)) and the measurement model (Eq. (8)).

The standard Kalman filter is only applicable to produce the estimation of true state $r(t)$ for each node individually. Nonetheless, all $m$ nodes measure the same dynamic process described in Eq. (7) and their estimation can be better calibrated once their measurements are shared among the network.

The Kalman-consensus information filter (KCIF) [54] is a decentralized form of Kalman filter to collaboratively estimate the targeted process $r(t)$ with better consensus. In particular, besides the standard Kalman estimator operations, each node will exchange messages among its neighboring nodes and enforce a consensus term on locally prior estimates to reach a consensus among all nodes. The Kalman-consensus information filter can be written as

$$
\hat{x}_{i}(t)=\bar{x}_{i}(t)+M_{i}(t)\left(y_{i}(t)-Y_{i} \bar{x}_{i}(t)\right)+C_{i}(t) \sum_{j \in N_{i}}\left(\bar{x}_{j}(t)-\bar{x}_{i}(t)\right),
$$

where $y_{i}(t)$ and $Y_{i}$ are weighted measurement and information matrix of neighbouring nodes of $i$, respectively, $N_{i}$ refers to the set of one-hop neighbors of node $i, M_{i}(t)$ is the posterior estimation covariance, and $C_{i}(t)$ is the consensus gain, which keeps the balance between the consensus and the stability of distributed Kalman estimators.

\section{OUR APPROACHES}

In this section, we first give a non-private solution for real-time decentralized statistical estimation for crowd-sourced data. Then, we detail our baseline solution with DP and communication efficiency, which is called DPCrowd for onedimensional and finite streams. Finally, we present the en-

\begin{tabular}{|c|c|}
\hline$m, d, T$ & Number of distributed servers, regions, timestamps \\
\hline$i, t, j, k$ & Index of servers, time, neighbouring servers, regions \\
\hline $\mathrm{SP}_{i}$ & $i^{\text {th }}$ distributed server \\
\hline$u_{i}^{j}$ & $j^{\text {th }}$ registered user at $\mathrm{SP}_{i}$ \\
\hline $\mathcal{E}(t)$ & Dynamic adjacent matrix at time $t$ \\
\hline$N_{i}$ & Neighboring set of $\mathrm{SP}_{i}$ \\
\hline $\bar{N}$ & Average node degree of distributed servers \\
\hline$r(t)$ & Overall real-time statistics at time $t$ \\
\hline$Q$ & Variance (covariance) of $r(t)$ \\
\hline$H_{i}$ & Observation coefficient of $\mathrm{SP}_{i}$ \\
\hline$f(\cdot)$ & Aggregate statistics function \\
\hline$x_{i}(t), z_{i}(t)$ & aggregate, and perturbed statistics of $\mathrm{SP}_{i}$ at time $t$ \\
\hline$R_{i}, \hat{R}_{i}$ & Variance (covariance) of observation statistics of $\mathrm{SP}_{i}$ \\
\hline $\bar{x}_{i}(t), \widehat{x}_{i}(t)$ & Prior/Posterior estimated statistics of $\mathrm{SP}_{i}$ at time $t$ \\
\hline$M_{i}(t)$ & Variance (covariance) of posterior estimation at $t$ \\
\hline$K_{i}(t), C_{i}(t)$ & Kalman/Consens gain of $\mathrm{SP}_{i}$ at time $t$ \\
\hline$P_{i}$ & Variance (covariance) of prior estimation at $\mathrm{SP}_{i}$ \\
\hline$u_{i}(t)$ & Weighted measurement of $\mathrm{SP}_{i}$ \\
\hline$y_{i}(t)$ & Average measurement of $\mathrm{SP}_{i}$ 's neighbours \\
\hline$U_{i}$ & Information matrix of $\mathrm{SP}_{i}$ \\
\hline$Y_{i}$ & Fused information matrix of $\mathrm{SP}_{i}$ 's neighbours \\
\hline
\end{tabular}
hanced solution, called DPCrowd+ for multi-dimensional and infinite streams. The main notations are listed in Table I.
TABLE I: Notations

\section{A. Non-private Solution}

One natural solution is that each distributed server independently estimates true statistics from its own database $D_{i, t}$.

1) Basic Idea: Without loss of generality, we simply denote the one-dimensional true statistics $r^{k}(t)$ at the $k^{t h}$, (where $\left.k=1, \ldots, d\right)$ dimension as $r(t)$. Then, we model $r(t)=f\left(D_{t}\right)$ as a dynamic process defined as Eq. (7), where $A(t)$ is the transition coefficient and can be simplified as a constant $A(t)=A=1$ when the timestamp is short. $\omega(t)$ is the process noise and follows a normal distribution, i.e., $\omega(t) \sim N(0, Q)$. Here, $Q$ can be learned from history data.

Since the user group $G_{i}(t)$ of each distributed server at slot $t$ can be regarded as a uniform sample of the whole population, it can be naturally assumed that the aggregate statistics $x_{i}(t)=f\left(D_{i, t}\right)$ at the server $\mathrm{SP}_{i}$ is an observation of the true time-series statistics $r(t)=f\left(D_{t}\right)$ and follows the linear equation as Eq. (8). The linear observation coefficients $H_{i}(t)$ corresponds to the ratio of the registered users $G_{i}(t)$, which represents the estimation weight of each distributed server in the crowd-sensing scenarios.

$$
H_{i}(t)=\frac{\left|G_{i}(t)\right|}{n},
$$

and $v_{i}(t)$ is the observation (measurement) noise and follows a normal distribution, i.e., $v_{i}(t) \sim N\left(0, R_{i}(t)\right)$. Since the uniform sample, there is generally, $R_{i}(t)=\left(H_{i}(t)\right)^{2} Q$.

To have an estimation for the real-time true statistics, the standard Kalman filter [24] or other temporal correlation exploitation techniques [9], [36], [35] can be adopted by each distributed server individually to exploit the temporal correlations in the aggregation data of crowd-sourced users, which can be formulated as Eq. (9). Nonetheless, due to independent estimation with partial knowledge, the estimations can be rather rough and no consensus can be achieved among distributed servers without mutual trust. An alternative solution is to multi-hop broadcast (i.e., blind flooding) each server's independent estimation to all others and then conduct weighted average estimation at all distributed servers. Nonetheless, the multi-hop broadcast would cause not only all-to-all communication complexity of $O\left(\mathrm{~m}^{2}\right)$, but also large estimation delay 
of at most $O(m)$ relays.

2) KCIF Based Statistical Estimation: We now propose a communication-efficient solution by utilizing Kalmanconsensus information filter [54] to collaboratively estimate the true statistics for distributed servers via only single-hop message exchange. The key idea is that each distributed server corrects its prior estimation with not only the standard Kalman process, but also the consensus information from its one-hop neighboring servers.

Algorithm 1 presents the KCIF based statistical estimation. At each timestamp, given initialized estimation covariance $P_{0}$ and prior estimation $\bar{x}_{i}(t)$, each server begins by obtaining its aggregate statistics $z_{i}(t)=f\left(D_{i, t}\right)$ from its partial crowd-sourced data $D_{i, t}$. Then, it computes and broadcasts the prior estimation, the weighted information vector $u_{i}(t)$ and matrix $U_{i}(t)$ to its one hop neighbors. Meanwhile, it receives the similar information from its direct neighbors $N_{i}$ and fuses the information as $y_{i}(t)=\sum_{j \in J_{i}} u_{j}(t)$, $Y_{i}(t)=\sum_{j \in J_{i}} U_{j}(t)$ (where $J_{j}=N_{j} \bigcup\{i\}$ ). After that, it computes the posterior estimation error covariance $M_{i}(t)$ and consensus gain $C_{i}(t)$ to derive the posterior estimation $\widehat{x}_{i}(t)$. Finally, it updates both the prior estimation and prior estimation covariance for the next iteration. With only onehop communications, all servers can collaboratively estimate the true statistics from their own partial database $D_{i}$ in a nonprivate way.

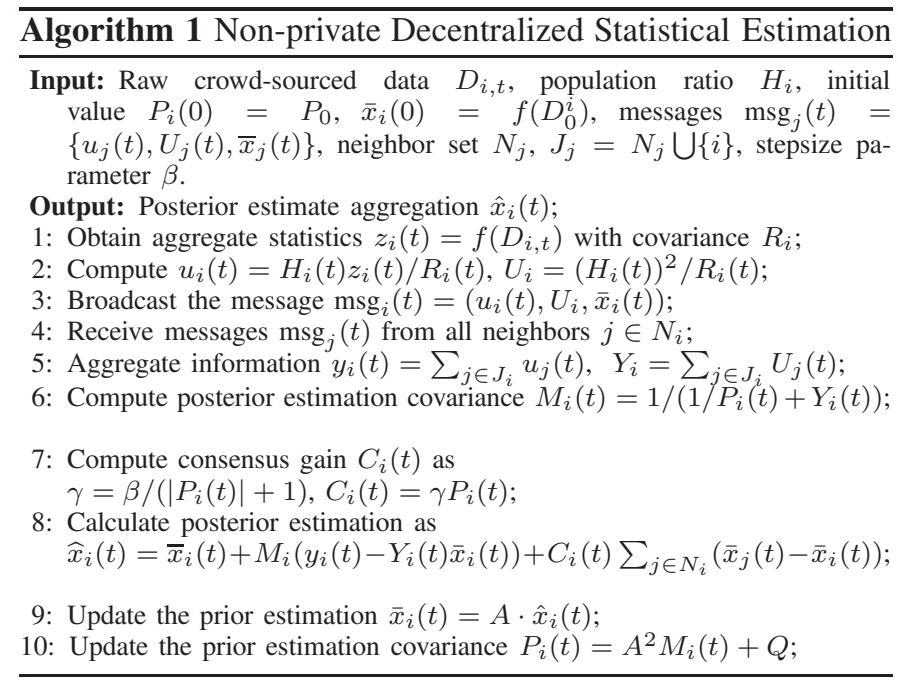

3) Challenges for DP and Communication-efficiency: The messages exchanged among servers in Algorithm 1 contains the information derived from the raw aggregate statistics $z_{i}(t)$, which may lead to the privacy exposure of individual users in $G_{i}$. Especially, servers may be geographically far away and not privacy reliable to each other. Besides, despite only single-hop communication, the continuous message exchange in Algorithm 1 at each timestamp would still incur great communication cost on a long timescale. To address both the concerns of privacy and communication cost, we aim to propose a real-time decentralized statistical parameter estimation framework with both DP and communication efficiency for multiple distributed servers on crowd-sourced data. A naive solution for DP suggests adding Laplace noise to the raw aggregate statistics $z_{i}$. However, we are still facing the following challenges:

- How to make the decentralized statistical estimation work in both communication-efficient and DP way?

- How to improve the estimation utility with given DP requirements (i.e., user-level $\varepsilon-D P$ for finite streams or $w$ event level DP for infinite streams) considering dynamic aggregation consumes DP budget quickly?

- How to reduce the estimation error for sparse regions in multi-dimensional data considering the DP noise may overwhelm the statistics over sparse regions?

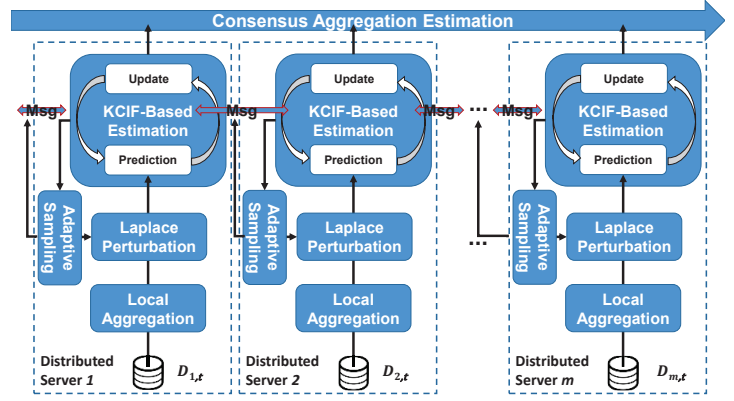

Fig. 2: A High-level Overview of DPCrowd

\section{B. DPCrowd: Real-time Decentralized Statistical Estimation for One-dimensional and Finite Data Streams}

To mitigate the privacy and communication challenges in the non-private solution of Section V-A, we first propose a baseline scheme DPCrowd with user-level $\varepsilon$-DP and communication-efficiency for real-time decentralized statistical estimation on one-dimensional and finite data streams.

1) Overview of DPCrowd: Fig. 2 presents a high-level overview of DPCrowd on distributed servers. DPCrowd mainly consists of three mechanisms: Laplace Perturbation, KCIF-based Estimation, and Adaptive Sampling based Intermittent Communication.

- Laplace Perturbation. After confirming $D_{i, t}$, each server computes the raw aggregate statistics $x_{i}(t)=$ $f\left(D_{i, t}\right)$ at time $t$. Here, we focus on estimating the one-dimensional statistics, e.g., the population of users appeared in a particular region. To guarantee user-level $\varepsilon$-DP for the finite stream, each server perturbs its raw statistics $x_{i}$ by Laplace mechanism with certain portion of allocated privacy budget, performs the post-process on perturbed statistics, and forwards the results to its neighbors.

- KCIF-based Estimation. KCIF-based estimation mechanism over each distributed server fuses the information exchanged from other servers and correct its own prior prediction according to both Kalman gain and consensus gain. Kalman gain can reduce both the observation noise and perturbation noise by exploiting the temporal correlations in real-time statistics. Consensus gain can integrate partial statistics from distributed servers to further correct overall estimation with consensus.

- Adaptive Sampling based Intermittent Communication. To reduce the communication cost and better utilize the privacy budget for a finite stream, we propose 
an adaptive sampling based intermittent communication strategy via leveraging the temporal correlations in crowd-sourced data. In particular, based on the dynamic changes between the prior estimation and posterior estimation after KCIF-based estimation, the server adaptively decides whether to perturb the aggregate statistics with certain privacy budget or approximate it with the previous estimation. Thus, the limited privacy budget can be allocated more to the necessary timestamps. Once the approximation strategy is chosen at the current timestamp, the server does not need to broadcast its estimations, thus further reducing the communication overheads.

Based on the above design rationales, Algorithm 2 presents the main procedures of DPCrowd at a distributed server $\mathrm{SP}_{i}$. In the following, we describe the main components with detailed procedures.

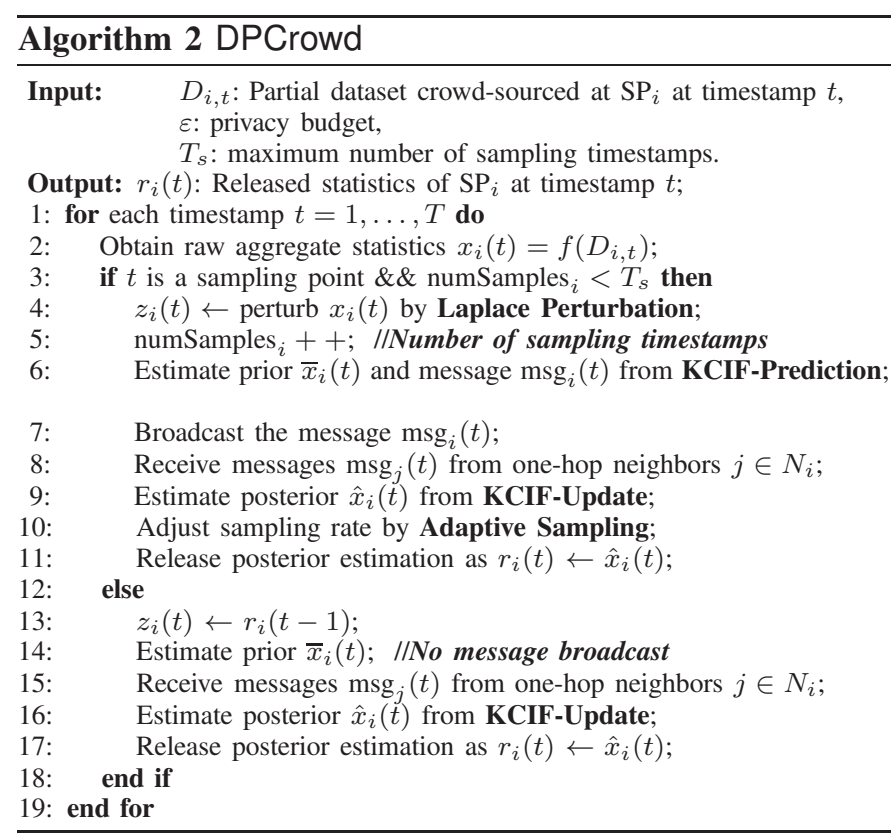

2) Laplace Perturbation: To realize user-level $\varepsilon$-DP at each server, the basic idea is to apply Laplace mechanism with different budget $\varepsilon(t)$ to inject Laplace noise to aggregate statistics at each time $t$, while keeping the total privacy budget consumption $\sum \varepsilon(t)$ for the finite stream no more than $\varepsilon$.

(1) Local Data Aggregation: At each timestamp $t$, the server $\mathrm{SP}_{i}$ obtains its aggregate statistic from its local crowdsourced data (i.e., $x_{i}(t)=f\left(D_{i, t}\right)$ ) and calculates its current observation coefficient $H_{i}(t)=\frac{\left|G_{i}(t)\right|}{n}$.

(2) Aggregate Data Perturbation: We adopt the Laplace mechanism to perturb the aggregate statistic $x_{i}(t)$ with a noise $v_{i}(t)$ drawn from the Laplace distribution $\mathcal{L}\left(\Delta_{f} / \varepsilon(t)\right)$, where $\Delta f$ is the sensitivity of the aggregate function $f(\cdot)$ and $\varepsilon(t)$ is the DP budget allocated at current timestamp. Then, we can obtain a noisy statistical value $z_{i}(t)=x_{i}(t)+v_{i}(t)$, which satisfies $\varepsilon(t)$-DP.

Particularly, taking the population sum of a region as the statistic function, since each crowd-sourcing user is associated with one distributed server at the same time and whether an individual user appears at a certain region can change $x_{i}(t)$ by at most 1 , the sensitivity of the statistic function is then $\Delta_{f}=1$. The Laplace noise $v_{i}(t)$ is drawn from Laplace distribution $\mathcal{L}(1 / \varepsilon(t))$, where $\varepsilon(t)$ is the DP budget at time $t$. For a time-series $x_{i}(t)$ with the time length of $T$, according to the sequential composition theorem, the privacy budget can be simply allocated as $\varepsilon(t)=\varepsilon / T$ at each time $t$ to meet the requirement of user-level $\varepsilon$-DP. However, much smaller $\varepsilon(t)$ would lead to larger amplitude of noise and worsened utility. Therefore, instead of uniform allocation of privacy budget $\varepsilon(t)=\varepsilon / T$ in the finite stream, we adaptively perturb the statistics at different timestamps by allocating different privacy budget according to the dynamic changes of $x_{i}(t)$ as described in Section V-B1. The detailed adaptive privacy budget allocation scheme will be introduced later in Section V-B4.

Remark 1. We make an assumption that each crowdsourcing user can be associated with only one distributed server at the same time in the system model of Section III. Without loss of generality, this assumption can be relaxed as each user can be associated with at most $c$ distributed servers at the same time. In such a case, the sensitivity can be set as $\Delta_{f}=c$ to increase the amount of perturbation noise in our algorithms to provide sufficient privacy preservation for any crowd-sourcing user.

Remark 2. Our work emphasizes designing a privacypreserving mechanism with a given parameter $\varepsilon$ while improving data utility. However, some work [50], [52] argued that DP on correlated data could offer $\varepsilon^{\prime}$-DP (where $\varepsilon<\varepsilon^{\prime} \ll T \varepsilon$ for general correlations), a weaker privacy guarantee when adopting the personal data principle [49], [50] in the different understanding of privacy [51]. Then, the privacy parameter $\varepsilon$ can be simply scaled down (by no more than $T$ times) according to the temporal correlation degree in the statistical results to satisfy stronger privacy protection under the personal data principle.

Combining with the original observation process in Eq. (8), the noisy local statistics $z_{i}(t)$ can be further represented as

$$
z_{i}(t)=H_{i}(t) r(t)+v_{i}(t)+v_{i}(t)=H_{i}(t) r(t)+o_{i}(t) .
$$

Here, $o_{i}(t)$ denotes the overall observation noise at $t$, which equals to the sum of two independent noise: the original observation noise $v_{i}(t)$ with the variance $\operatorname{Var}\left(v_{i}(t)\right)=2\left(\frac{\Delta_{f}}{\varepsilon(t)}\right)^{2}$, and the privacy-preserving noise $v_{i}(t)$ with the variance $\operatorname{Var}\left(v_{i}(t)\right)=\left(H_{i}(t)\right)^{2} Q$. Then, $z_{i}(t)$ can be further postprocessed and shared with other servers to jointly estimate the true statistics $r(t)$ later.

3) KCIF-Based Estimation: To collaboratively estimate the true statistics with consensus, each server not only needs to conduct prior estimation according to its own knowledge, but also corrects the prior estimation via messages exchange. Based on the non-private solution in Section V-A, we adopt a KCIF-based estimation mechanism to fuse the information from distributed servers, thus achieving both high utility and consensus.

(1) Noise Model of Kalman Filter: Generally speaking, Kalman filter achieves the optimal posterior estimation when the measurement noise follows the Gaussian distribution. Fortunately, as proved in [24], Kalman filter works effectively 
on the noise with Laplace distribution $\mathcal{L}\left(\Delta_{f} / \varepsilon(t)\right)$ when the variance parameter $R$ in Kalman filter satisfies $R \propto 2\left(\frac{\Delta_{f}}{\varepsilon(t)}\right)^{2}$. That is to say, we can use a Gaussian distribution $\mathcal{N}(0, R)$ to approximate the Laplace distribution $\mathcal{L}\left(\Delta_{f} / \varepsilon(t)\right)$ for privacy preservation. Thus, according to Eq. (12), to achieve minimum variance posterior estimate under both the observation noise and the privacy-preserving noise, the optimal value $\hat{R}_{i}(t)$ for Kalman filter can be set as

$$
\hat{R}_{i}(t) \propto \alpha \cdot 2\left(\frac{\Delta_{f}}{\varepsilon(t)}\right)^{2}+\left(H_{i}(t)\right)^{2} \cdot Q,
$$

where $\alpha$ is an adjustable proportional coefficient. This approximation has also been verified in our experiments in Section VII.

(2) KCIF-Prediction: KCIF-Prediction maintains a prior estimation $\bar{x}_{i}(t)$ for each server $\mathrm{SP}_{i}$. It can be initialized as

$$
\bar{x}_{i}(0)=x_{i}(0) / H_{i}(0) .
$$

After that, according to Eq. (7), the prior estimation can be predicted as its previous estimation.

$$
\bar{x}_{i}(t)=A \hat{x}_{i}(t-1) \text {. }
$$

In addition, according to the standard Kalman filter, the prior estimation error covariance $P_{i}(t)$ of $\mathrm{SP}_{i}$ can be predicted as

$$
P_{i}(t)=A^{2} M_{i}(t-1)+Q,
$$

where $M_{i}(t-1)$ is the posterior error covariance at time $t-1$ and $Q$ is the variance of process noise in Eq. (7). The posterior error covariance can be initialized as $M_{i}(0)=$ $\left(H_{i}(t)\right)^{2} / \hat{R}_{i}(t)$, where $\hat{R}_{i}(t)$ is set according to Eq. (13).

(3) Message Exchange: After perturbation and prediction, each server exchanges messages with their neighbors in one hop for collaborative estimation. The message $\operatorname{msg}_{i}(t)$ encapsulated from $\mathrm{SP}_{i}$ consists of three parts: the prior estimation $\bar{x}_{i}(t)$, the weighted statistics $u_{i}(t)$, and the information matrix $U_{i}(t)$. In particular, $u_{i}(t)$ and $U_{i}(t)$ can be computed as

$$
\begin{aligned}
u_{i}(t) & =\left(H_{i}(t) \cdot z_{i}(t)\right) / \hat{R}_{i}(t), \text { and } \\
U_{i}(t) & =\left(H_{i}(t)\right)^{2} / \hat{R}_{i}(t) .
\end{aligned}
$$

After that, $\operatorname{msg}_{i}(t)=\left(\bar{x}_{i}(t), u_{i}(t), U_{i}(t)\right)$ is broadcasted to the directed neighbors. $\operatorname{msg}_{i}(t)$ only contains sanitized information of $\mathrm{SP}_{i}$ 's aggregate statistics over $D_{i, t}$, which do not leak the privacy.

(4) KCIF-Update: Receiving the messages from direct neighbors $j \in N_{i}, \mathrm{SP}_{i}$ first sums up the weighted aggregation $u_{j}(t)$ and the weighted information matrices $U_{j}(t)$ as follows.

$$
\begin{aligned}
y_{i}(t) & =\sum_{j \in N_{i} \bigcup\{i\}} u_{j}(t), \\
Y_{i}(t) & =\sum_{j \in N_{i} \bigcup\{i\}} U_{j}(t) .
\end{aligned}
$$

Then, combining with the prior estimation error covariance $P_{i}, \mathrm{SP}_{i}$ will compute both the posterior estimation error covariance $M_{i}(t)$ and consensus gain $C_{i}(t)=\gamma_{i}(t) P_{i}(t)$ in Kalman-consensus information filter, respectively.

$$
\begin{aligned}
M_{i}(t) & =1 /\left(1 / P_{i}(t)+Y_{i}(t)\right), \\
C_{i}(t) & =\gamma_{i}(t) P_{i}(t)=\beta P_{i}(t) /\left(\left|P_{i}(t)\right|+1\right),
\end{aligned}
$$

where $\beta>0$ is a relative small constant with the order of the time step size in discretization of the continuous time process.

Finally, according to the Kalman-consensus information filter, the posterior estimation $\hat{x}_{i}(t)$ at $\mathrm{SP}_{i}$ can be computed as

$$
\begin{aligned}
& \hat{x}_{i}(t)=\bar{x}_{i}(t)+ \\
& M_{i}(t)\left(y_{i}(t)-Y_{i}(t) \bar{x}_{i}(t)\right)+C_{i}(t) \sum_{j \in N_{i}}\left(\bar{x}_{j}(t)-\bar{x}_{i}(t)\right) .
\end{aligned}
$$

With the correction of standard Kalman estimation term controlled by the posterior estimation error covariance $M_{i}(t)$ and the consensus term controlled by consensus gain $C_{i}(t)=$ $\gamma_{i} P_{i}(t)$ in Eq. (23), the posterior estimations of true statistics at each distributed server will gradually reach both accuracy and consensus.

4) Adaptive Sampling based Intermittent Communication: According to the design rationale in Section V-B1, a sampling based intermittent communication strategy can provide the following benefits for DPCrowd:

- Communication Efficiency. Considering the signal sparsity in streams, despite only one-hop communication between neighboring servers, the continuous message exchanges at all timestamps of KCIF-based estimation in Section V-B3 seems to be communication expensive, in terms of the length $T$ of the finite data stream. One common method of communication reduction is to reduce the communication frequency via sampling.

- Privacy Budget Allocation. As described in Section V-B2,to achieve user-level $\varepsilon$-DP for a finite stream with the time length of $T$ timestamps, one simple idea is to uniformly allocate the total privacy budget $\varepsilon$ for all $T$ timestamps. Then, if $T$ is large, the average privacy budget $\varepsilon(t)$ used for each timestamp will be small and lead to the low utility of estimation at each server. To enhance the estimation accuracy, one key idea is to reduce the noise addition by selectively allocating more privacy budget at some sampling timestamps and approximating aggregation results at non-sampling timestamps with previous estimations without privacy budget consumption.

Combining the above ideas, we propose to apply the sampling based intermittent communication strategy to both reduce the communication frequency in KCIF-based estimation (Section V-B3) and save up the privacy budget in Laplace perturbation (Section V-B2), without significantly affecting the estimation utility. The basic idea is that, only at the sampling timestamps, each distributed server allocates privacy budget and sends DP protected messages to its one-hop neighbors (Lines 3 11 in Algorithm 2); while at the non-sampling timestamps, each distributed server approximates the prior estimation with previous posterior estimation without privacy budget and does not send out messages (Lines 12 17 in Algorithm 2).

One straightforward solution is the fixed-rate sampling strategy. Given a predefined sampling interval $I$, each server $\mathrm{SP}_{i}$ will periodically sample and perturb its aggregate statistics $x_{i}(t)$ by Laplace mechanism. The total number of sampling and communication timestamps for each server is $T_{s}=T / I$, 
and the privacy budget for each sampling timestamp $t$ is $\varepsilon(t)=\varepsilon / T_{s}$. The choice of sampling rate (or sampling interval $I)$ has the following impacts:

- When $I$ is small, the communication frequency is high with less consensus error, but $\varepsilon(t)$ is small and too many sampling timestamps will lead to much perturbation error.

- When $I$ is large, communication frequency and perturbation error can be reduced, but a large sampling gap will cause larger approximation and concensus error.

Thus, both sampling and non-sampling timestamps will cause errors and may have an impact on the overall accuracy. To achieve higher accuracy, it requires to seek the optimal sampling rate according to some prior knowledge about the data, which is, however, not applicable in real-time crowdsourced data. A good sampling strategy should adjust the sampling rate to minimize the two errors with given privacy budget $\varepsilon$. We apply the adaptive-rate sampling strategy based on PID control in FAST [24] to adjust the sampling rate based on the dynamics of the statistics. In particular, each server $\mathrm{SP}_{i}$ dynamically adjusts it own sampling intervals $I_{i}$ according to the real-time error between the prior and posterior estimations. The details can be referred to [24].

\section{DPCrowd+: Real-time Decentralized Statistical Estima- tion for Multi-dimensional and Infinite Data Streams}

So far, DPCrowd focuses on the crowd-sourced data with one-dimension and limited length, e.g., distribute servers only care about the true statistic of a particular region in a particular time period. Nonetheless, in reality, typical crowd-sourced data are multi-dimensional (even high-dimensional) and infinitely generated. For example, the servers need to estimate the true statistics over all regions uninterruptedly. In such a case, there are two challenges for DPCrowd:

- Without consideration of the sparsity, the same amount of noise would be added to all regions and ruin the utility of those regions with a small value.

- Simple event-level DP or user-level DP on finite streams will be not applicable to infinite data streams as the total privacy budget accumulates with the time.

To address the aforementioned challenges, we further propose DPCrowd+, a more applicable privacy-preserving decentralized statistical estimation mechanism for multi-dimensional infinite crowd-sourced data streams.

1) Data Modeling: Before introducing the details of DPCrowd+, we first extend the data model in Section V-B to a multi-dimensional scenario. Similar to DPCrowd, multidimensional true statistics $\mathbf{r}(t)$ can be modeled and formulated by vectors as follows

$$
\mathbf{r}(t+1)=\mathbf{A}(\mathbf{t}) \cdot \mathbf{r}(t)+\boldsymbol{\omega}(t),
$$

where $\mathbf{r}(t)$ are $d$-dimensional vector and each element $r^{k}(t)$ represents the true statistics of region $k$ at timestamp $t$. $\mathbf{A}(\mathbf{t})=\left[a_{i, j}(t)\right]_{d \times d}$ can be a $d \times d$ time-varying transition matrix, which models the correlations among dimensions (e.g., regions). Particular, matrix $A(t)$ at time $t$ may be a general linear transformation matrix or a Markov matrix (stochastic matrix). For a Markov matrix, each element $a_{i, j}(t)$ may represent the probability that a user may transit from region $i$ to $j$ in a city or from website $i$ to $j$ during Internet surfing at different time $t$ [55]. For simplicity, we consider $\mathbf{A}(\mathbf{t})=\mathbf{A}$ is a constant linear transition matrix, which can be trained from the history data.

Also, $\boldsymbol{\omega}(t)=\left(\omega^{1}(t), \omega^{2}(t), \ldots, \omega^{d}(t)\right)$ is the $d$-dimensional process noise that follows the $d$-dimensional Gaussian distribution, i.e., $\boldsymbol{\omega}(t) \sim \boldsymbol{N}(0, \mathbf{Q})$, where $\mathbf{Q}=\left[Q_{i, j}\right]_{d \times d}$ is the covariance matrix and each element $Q_{i, j}$ is a scalar value and represented as the covariance of $\omega_{d}(t)$. Although the constant matrix $\mathbf{A}(\mathbf{t})$ represents the general steady correlations among dimensions, the time-varying process noise $\boldsymbol{\omega}(t)$ can reflect the dynamic changes of dimensional correlations and sparsity. For example, the unusual social events may lead to the changes of traffic patterns or webpage views at a certain period. For simplicity, we assume that the process noise of each dimension is independent of each other, i.e., $Q_{i, j}=0, i \neq j$, then its covariance matrix $Q$ can be simplified as

$$
\mathbf{Q}=\operatorname{diag}\left(Q_{1,1}, Q_{2,2}, \ldots, Q_{d, d}\right) .
$$

Meanwhile, we assume the aggregate $d$-dimensional statistical vector $\mathbf{x}_{i}(t)$ at each distributed server also follows a linear equation as

$$
\mathbf{x}_{i}(t)=H_{i}(t) \cdot \mathbf{r}(t)+\mathbf{v}_{i}(t),
$$

where $H_{i}(t)$ at slot $t$ is a scalar value represents the linear observation coefficients as Eq. (11) and $\mathbf{v}_{i}(t)=$ $\left(v_{i}^{1}(t), v_{i}^{2}(t), \ldots, v_{i}^{d}(t)\right)$ is a $d$-dimensional observation noise. We assume that each element of $\mathbf{v}_{i}(t)$ is independent from each other and follows the zero-mean Gaussian distribution with the variance $\mathbf{R}_{i}(t)=\left(H_{i}(t)\right)^{2} \mathbf{Q}$. Then, there is $v_{i}^{k}(t) \sim$ $N\left(0, R_{i}^{k}(t)\right)$, where $R_{i}^{k}(t)=\left(H_{i}(t)\right)^{2} Q_{k, k}(k=1,2, \ldots, d)$.

2) DPCrowd+ based on Dynamic Grouping: The workflow of DPCrowd+ on each distributed server is shown in Fig. 3. Compared with DPCrowd shown in Fig. 2, DPCrowd+ includes two more components: (i) dynamic grouping and (ii) adaptive budget allocation, inspired by [9]. In the dynamic grouping mechanism, similar regions with small values will be grouped together to avoid the overdose of noise. In particular, the correlations of different regions are calculated based on the previously published results to guarantee privacy. Thus, highdimensional aggregate statistics may be grouped into several groups and different Laplace noise is then added to each group to strike a good balance between privacy and utility. The adaptive budget allocation mechanism is responsible for allocating the privacy budget to make sure $w$-event DP is satisfied in the infinite aggregation stream. Thus, besides adopting adaptive sampling to reduce the noise, the privacy budget for each timestamp should be carefully allocated to meet the requirement. The privacy budget will be allocated according to the dynamics of grouped regions to improve the utility. Since the dynamic grouping and adaptive allocation mechanism are exactly the same as those in RescueDP algorithm. Please refer to [9] for more detail.

Algorithm 3 presents the main procedures of DPCrowd+. It should be noted, other components, i.e., Laplace perturbation, KCIF-based estimation, adaptive sampling will also be 


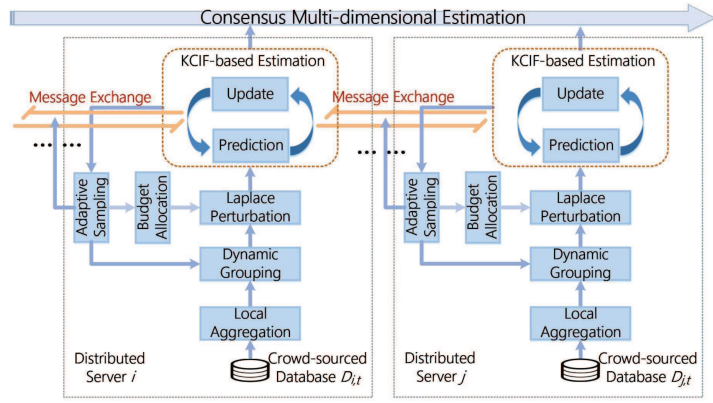

Fig. 3: A Framework of DPCrowd+

adjusted to incorporate dynamic grouping and adaptive budget allocation.

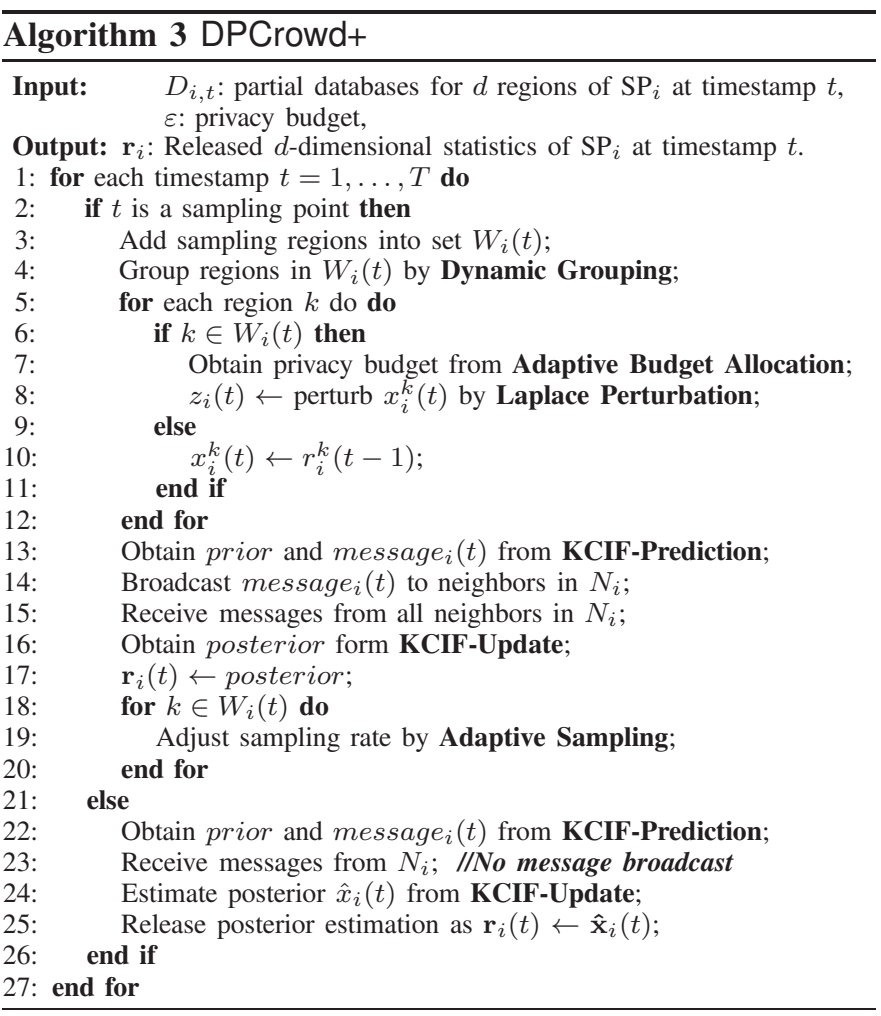

\section{Algorithm Analysis}

In this section, we conduct the theoretical analysis of our scheme in terms of privacy protection and utility, as well as communication latency and cost.

\section{A. Privacy Analysis}

Theorem 4 DPCrowd in Algorithm 2 guarantees user-level $\varepsilon-D P$ for the registered crowd-sourcing users for a finite stream with the length of time $T$ at each server $S P_{i}$.

Proof Given the maximum number of sampling timestamps $T_{s}$ and the total privacy budget $\varepsilon$, each Laplace perturbation adds noise drawn from the Laplace distribution $\mathcal{L}\left(\Delta f \cdot T_{s} / \varepsilon\right)$ at each sampling timestamp, which satisfies $\varepsilon / T_{s}-D P$ for each crowd-sourcing user according to the Theorem 1. Then, based on Theorem 2, after $T_{s}$ sampling timestamps, Laplace perturbations on the aggregate statistics satisfies $\varepsilon-D P$ for each user for the whole finite stream.

Among all processes in DPCrowd, only the process of Laplace perturbation can access to the true aggregate statistic at each timestamp at each server, and other processes are all conducted on the perturbed statistics. Thus, according to the post-processing property in Theorem 3, DPCrowd satisfies user-level $\varepsilon-D P$ for the finite stream with length $T$ at each server.

Theorem 5 DPCrowd+ in Algorithm 3 guarantees w-event $\varepsilon-D P$ for the registered crowd-sourcing users for an infinite stream at each distributed server $S P_{i}$.

Proof Similar to Proof of Theorem 4, we prove DPCrowd+ satisfies w-event $\varepsilon-D P$ if and only if the Laplace perturbation satisfies w-event $\varepsilon-D P$. In particular, according to [9], the perturbed statistics satisfy w-event $\varepsilon-D P$, our adoption of multidimensional Laplace mechanism with both dynamic grouping and adaptive privacy budget allocation strategies would satisfy $w$-event $\varepsilon$-DP for each region $k$ at each server $S P_{i}$. Therefore, $D P C r o w d+$ satisfied w-event $\varepsilon-D P$.

\section{B. Utility Analysis}

Without loss of generality, we mainly focus on the general error analysis of DPCrowd for one-dimensional data streams (DPCrowd+ can have similar conclusions) without considering the adaptive sampling mechanism. The mean square posterior estimation error of $\mathrm{SP}_{i}$ at timestamp $t$ can be calculated as

$$
\mathbb{E}\left[\left|\hat{x}_{i}(t)-x(t)\right|^{2}\right]=\mathbb{E}\left[\left(\hat{x}_{i}-x(t)\right)\left(\hat{x}_{i}-x(t)\right)\right],
$$

which is also equal to the error variance matrix $M_{i}(t)$ for one-dimensional data (or the trace of the error covariance matrix for multi-dimensional data) of $\mathrm{SP}_{i}$ according to Kalman consensus information filter [54]. Based on Algorithms 1 and 2 , for one-dimensional case, we have

$$
\begin{aligned}
M_{i}(t) & =1 /\left(1 / P_{i}(t)+Y_{i}(t)\right) \\
& =1 /\left(1 / P_{i}(t)+\left(H_{i}(t)\right)^{2} / \hat{R}_{i}(t)\right), \\
& =\frac{\hat{R}_{i}(t) P_{i}(t)}{\hat{R}_{i}(t)+\left(H_{i}(t)\right)^{2} P_{i}(t)} \\
& =\frac{\hat{R}_{i}(t)\left(M_{i}(t-1)+Q\right)}{\hat{R}_{i}(t)+\left(H_{i}(t)\right)^{2}\left(M_{i}(t-1)+Q\right)},
\end{aligned}
$$

where $M_{i}(t)$ is initialized as $M_{i}(0)=\left(H_{i}(0)\right)^{2} / \hat{R}_{i}(0)$. From Eq. (28), we can have the following observations about the estimation error.

- The posterior estimation error is decided by the observation noise variance $\hat{R}_{i}(t)$ (including that caused by privacy-preserving noise), the process noise variance $Q$, and the coefficient $H_{i}(t)$.

- With the increase of $\varepsilon$, observation noise variance $\hat{R}_{i}(t)$ decreases, and so does the error variance. This also shows the general trade-off between utility and privacy in DPCrowd.

- As $\hat{R}_{i}(t)$ is the same order of $Q$, the posterior estimation error would increase with process noise variance $Q$. This 
implies the stream of statistics with more fluctuates (i.e., larger $Q$ ) would generally cause larger estimation error.

- Since $M_{i}(0)$ is iteratively substituted in the update of $M_{i}(t)$, it is not difficult to see that the posterior estimation error would become small when the coefficient $H_{i}(t)$ is large.

\section{Communication Latency and Cost}

In both DPCrowd and DPCrowd+, each distributed server $\mathrm{SP}_{i}$ only exchanges messages with its one-hop neighbors at each timestamp. The communication latency is only $O(1)$ hop and the communication complexity is equal to $O\left(\sum_{i=1}^{m}\left\|N_{i}\right\|\right)=$ $O(m \cdot \bar{N})$ in terms of the number of distributed servers $m$ (network scale), where $\left\|N_{i}\right\|$ is the degree (or the cardinality of neighboring set $N_{i}$ ) of $\mathrm{SP}_{i}$, and $\bar{N}$ is the average node degree of the network. Assuming that $\bar{N}=O(\log (m))$, the communication cost of both DPCrowd and DPCrowd+ is then $O(m \log (m))$, which is scalable in terms of the number of distributed servers $m$.

According to the adaptive sampling based intermittent communication in Section V-B4, each server only incurs message broadcasts at its sampling timestamps. In DPCrowd, suppose that the total timestamp length is $T$, then for the fixed sampling strategy, given the sampling interval $I$, the communication reduction ratio is $I / T$; while for the adaptive sampling strategy, given the maximal sampling timestamps of $T_{s}$, the communication reduction ratio is $T_{s} / T$.

\section{Performance Evaluation}

We conducted extensive experiments on both synthetic and real-world datasets to demonstrate both the effectiveness and efficiency of our proposed algorithms DPCrowd and DPCrowd+.

\section{A. Simulation Setup}

Datasets: For single-dimensional data, we used one synthetic dataset and two real-world datasets as follows:.

- Linear is a synthetic dataset consisting of 1000 timestamps, which are generated according to the process model in Eq. (7) with the variance $Q$ as $10^{5}$.

- Flu ${ }^{1}$ is part of the weekly surveillance data of flu infection provided by the Influenza Division of the Center for Disease Control and Prevention. We extracted a timeseries consists of 791 timestamps for each weekly report.

For multi-dimensional data, we also used one synthetic dataset and one real-world dataset.

- Multi-Linear is synthesized according to the process model of Eq. (24). It is a six-dimensional time-series with 1000 timestamps. The size of both the transition matrix $\boldsymbol{A}$ and covariance matrix $\boldsymbol{Q}$ is $6 \times 6$.

- Multi-Flu is a multi-dimensional version of Flu and contains the weekly outpatient death population of 51 states in US for 441 weeks between 2009 and 2017.

\footnotetext{
${ }^{1}$ http://www.cdc.gov/flu
}

We approximated the transition matrix $\boldsymbol{A}$ by frequency statistics and trained the optimal covariance matrix $Q$ by genetic algorithm, in which the average relative error is used as the input of fitness function.

Simulation Methodology: We implemented all algorithms in Matlab for simulating the interactions among $m=50$ distributed servers in a fully decentralized network. The network topology is described by an evolving stochastic matrix $\mathcal{E}(t)$, which is randomly generated with various level of network density. Based on the network model in Section III, the network density $\rho$ is defined as

$$
\rho=\frac{2 * \text { num }_{E}}{m(m-1)},
$$

where $m$ is the number of distributed servers and num $_{E}$ is the average number of edges in $\mathcal{E}(t)$. The communication latency between any two servers is assigned as a random number follows a uniform distribution around $100 \mathrm{~ms}^{2}$. Besides, each distributed server is assigned a random observation coefficient of $H_{i}(t)$ sampled according to uniform distribution $U(0,1)$. Finally, each server fuses the received data to correct its posterior estimation. The above processes are repeated until all timestamps of each dataset are touched.

Comparison: To show the effectiveness of our schemes, we also summarized, simulated and compared with the coral algorithms of relevant and typical benchmark schemes on differentially private streaming: FAST [24], RescueDP [9], BD/BA [36], and PeGaSus [35]. We extended them to realize real-time decentralized statistical estimation by the straightforward whole-network broadcasting and averaging, discussed in Section V-A1. For simplicity, we denote these extension schemes as DFAST, DRescueDP, DBD/DBA, and DPeGaSus, respectively. To fairly compare the utility, we also further extended DBD/DBA, and DPeGaSus to support multi-dimensional data streams and transformed DPeGaSus to meet the equivalent $w$-event level privacy level. For example, we extended $w$-event level BD/BA and event-level PeGaSus to meet the same $w$-event level privacy guarantee for DPCrowd or $w$-event level privacy for DPCrowd+. We also extended our DPCrowd into $\mathrm{DPCrowd}_{w}$ to compare the utility improvement of DPCrowd+ by applying the basic DPCrowd independently on each dimension and each $w$ timestamp-long non-overlapping window of an infinite multidimensional stream. The detailed features of the main comparable schemes are listed in Table II.

Moreover, we compared the performance of our schemes under different strategies and extensions mentioned before, such as the intermittent communication of DPCrowd framework under different sampling strategies (fixed-rate sampling vs. adaptive sampling).

Metrics: In terms of accuracy, we adopted the metric of average relative error (ARE) to measure the relative distance between the final estimation $\hat{x}_{i}(t)$ and the ground truth $r(t)$,

\footnotetext{
${ }^{2}$ The runtime of core algorithms are much faster, e.g., Line $2 \sim 17$ in Algorithm 2 (DPCrowd) and Line $2 \sim 25$ in Algorithm 3 (DPCrowd+) consume less than $0.1 \mathrm{~ms}$ when executed on a real machine (Matlab R2018a, Win10, 8GB RAM, CPU i5-5200U).
} 
TABLE II: Features of Main Comparable Schemes

\begin{tabular}{|l|c|c|c|c|}
\hline Schemes & Architecture & Communication & Dimension Correlation & Privacy Level \\
\hline FAST [24] & Centralized & No communication & Single dimension & user level \\
DFAST $[24]^{*}$ & Decentralized & Multi-hop $\times$ Continuous & Single dimension & user level \\
DRescueDP [9] & Decentralized & Multi-hop $\times$ Continuous & Correlated dimensions & $w$-event level \\
DBD $/$ DBA [36] & Decentralized & Multi-hop $\times$ Continuous & Correlated dimensions & $w$-event level \\
DPeGaSus [35] & Decentralized & Multi-hop $\times$ Continuous & Independent dimensions & $w$-event level \\
DPCrowd & Decentralized & One-hop $\times$ Intermittent & Single dimension & user level \\
DPCrowd $w$ & Decentralized & One-hop $\times$ Intermittent & Independent dimensions & $w$-event level \\
DPCrowd+ & Decentralized & One-hop $\times$ Intermittent & Correlated dimensions & $w$-event level \\
\hline
\end{tabular}

* DFAST, DRescueDP, DBD/DBA, DPeGaSus are the decentralized extension (via broadcasting and averaging at each server) of centralized schemes FAST [24], RescueDP [9], BD/BA [36], and PeGaSus [35], respectively

$1 \leq i \leq m, 1 \leq t \leq T$. The ARE is defined as

$$
\operatorname{ARE}(\hat{\boldsymbol{x}}, \boldsymbol{r})=\frac{1}{m} \frac{1}{T} \sum_{i=1}^{m} \sum_{t=1}^{T} \frac{\left|\hat{x}_{i}(t)-r(t)\right|}{\max (r(t), \delta)},
$$

where $\delta$ is set as 1 in case that $x(t)$ is 0 . As observed, smaller ARE means the estimation is more close to the ground truth and have better accuracy. With regard to consensus, we used the metric of average consensus error (ACE) to measure the closeness of estimations $\hat{x}_{i}(t)$ among distributed servers $1 \leq$ $i \leq m$. The ACE is defined as

$$
A C E=(\hat{\boldsymbol{x}})=\frac{1}{m} \frac{1}{T} \sum_{i=1}^{m} \sum_{t=1}^{T}\left|\hat{x}_{i}(t)-\hat{x}_{\text {average }}(t)\right|,
$$

where $\hat{x}_{\text {average }}(t)$ is average estimation of all distributed servers. Similarly, smaller ACE means better consensus among distributed servers. For a fair comparison, each set of experiments is run 50 times and the average result is reported.

Parameters: The default parameters and their descriptions, unless otherwise explained, are listed in Table III. In our simulations, we chose the optimal parameters by experimentally minimizing the posterior estimation error. For example, we first chose optimal model variance $Q$ for different datasets; the sampling parameters $M, \theta, \xi$ were chosen separately to minimize the final error; $R$ was approximated according to Eq. (13) and varied across datasets.

\section{B. Estimation Utility of DPCrowd}

Convergence of Estimation: Fig. 4 demonstrates the timevarying estimation error of DPCrowd among all distributed servers, in comparison with that of related schemes. In Fig. 4a, the relative error of distributed servers in all three schemes gradually drops with time. This is because, distributed servers would initially produce rough prior estimates, which are then gradually corrected via observing new measurements. Nonetheless, without communication, servers in FAST can only observe their own measurements and perform independent estimation, thus converging slowly with much higher consensus error. DFAST simply collects and averages the independent estimations in the whole network. Despite the reduced relative error via averaging, it still has the same convergence speed as FAST, which is determined by the independent estimation. Besides, although DFAST can achieve absolute consensus, it would lead to huge communication cost in blind flooding. Differently, distributed servers in DPCrowd conduct estimation via information fusion with their one-hop neighbors at each time, therefore shows fast convergence. With the increase of time, the estimations of all distributed servers will be finally disseminated and fused according to the weights to achieve both accurate and approximate consensus. Unlike Linear better follows an approximately linear process, Flu has more periodic fluctuations. Nonetheless, in Fig. 4b, DPCrowd still shows much better estimation convergence.

Impact of Network Density: Fig. 5 presents the impact of network density $\rho$ on both ARE and ACE of DPCrowd compared with DFAST. Since estimation results are broadcast to all servers, the ARE of DFAST remains unchanged for different $\rho$ and its ACE is as small as zero. On both datasets, both the ARE and ACE of DPCrowd decrease with $\rho$ since a denser network can better guarantee the convergence via more extensive communications. Both errors in the stronger privacy regime $(\varepsilon=0.1)$ are larger than those in the weaker privacy regime $(\varepsilon=1)$, which reflects the utility-privacy tradeoff. When $\varepsilon=1$, both ARE and ACE are not sensitive to $\rho$. This is because, with less noise, distributed servers can easily achieve accurate and consensus estimation with fewer neighbors. As shown, ARE and ACE of DPCrowd also vary across datasets. As analyzed before, unlike Linear, the higher ARE and ACE of DPCrowd on Flu result from the large fluctuations of both dataset.

Impact of Sampling Strategy: Fig. 6 reports the ARE and ACE of DPCrowd under both fixed and adaptive sampling based intermittent communication strategies. Because of the adaptiveness, DPCrowd-Adaptive keeps nearly the same error. Nonetheless, DPCrowd-fixed varies greatly with different sampling intervals. When the interval is small (such as 1), it incurs high perturbation error on both datasets because much noise is injected at nearly every timestamp. When the sampling interval increases slightly, it performs better since less noise is injected in a sampling manner. Nonetheless, with the further increase of intervals, the ARE of DPCrowd-fixed increases gradually and goes beyond that of DPCrowd-Adaptive since larger sampling interval will lead to larger prediction error in spite of smaller perturbation error. Similar trends can be observed in the ACE comparison. DPCrowd-fixed seems to show a smaller consensus error. The reason is that consensus error mainly comes from the perturbation error, which is much smaller when there are more non-sampling stamps. In other words, larger sampling interval means more non-sampling points and less dynamic changes, which naturally lead to better consensus, but less accuracy. Thus, ARE and ACE should be combined to analyze the performance of DPCrowd. Overall, DPCrowd under the adaptive sampling strategy is more robust to different datasets.

Tradeoff between Utility and Privacy: Fig. 7 compares both ARE and ACE of DPCrowd with FAST and DFAST under different privacy $\varepsilon$. All AREs decrease with $\varepsilon$, which demonstrates the trade-off between utility and privacy. However, the ARE of DPCrowd and DFAST is consistently lower than that of FAST as both schemes can greatly improve the estimation via communications. Furthermore, DPCrowd incurs less ARE than DFAST in most cases since the estimation can be better corrected according to the weights of different servers (Eq. 17). Compared with Fig. 7b, Fig. 7a has the lowest ARE 
TABLE III: Parameters Setup for Different Datasets in Experiments

\begin{tabular}{|c|c|c|c|c|c|}
\hline Symbol & Description & Linear & Flu & Multi-Linear & Multi-Flu \\
\hline$\varepsilon$ & Total Privacy Budget & 1 & 1 & 1 & 1 \\
$d$ & Data Dimensionality & 1 & 1 & 6 & 51 \\
$\left(C_{p}, C_{i}, C_{d}\right)$ & PID Control Gains & $(0.9,0.1,0)$ & $(0.9,0.1,0)$ & $(0.9,0.1,0)$ & $(0.9,0.1,0)$ \\
$T$ & Total Timestamps & 1000 & 791 & 1000 & 441 \\
$T_{i}$ & Integral Time Window & 5 & 5 & 5 & 5 \\
$T_{s}$ & Maximal Sampling Number & $0.3 T$ & $0.4 T$ & N/A & N/A \\
$(\theta, \xi)$ & Interval Adjustment Parameters & $(2.5,0.05)$ & $(1,0.6)$ & $(1, \mathrm{~N} / \mathrm{A})$ & $(3, \mathrm{~N} / \mathrm{A})$ \\
\hline
\end{tabular}

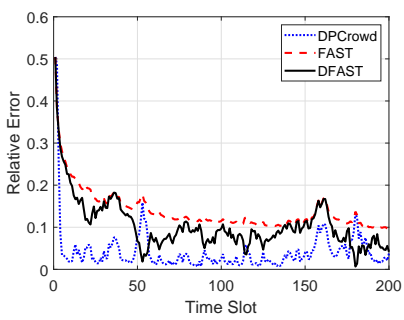

(a) Linear

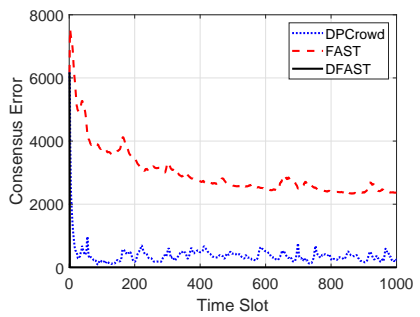

Fig. 4: Error Convergence with Time

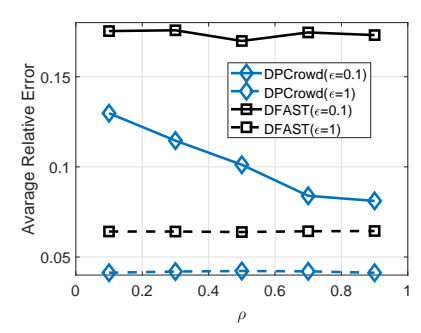

(a) Linear
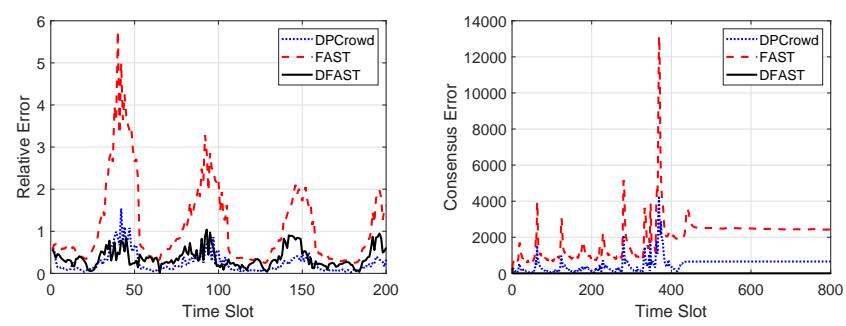

(b) Flu

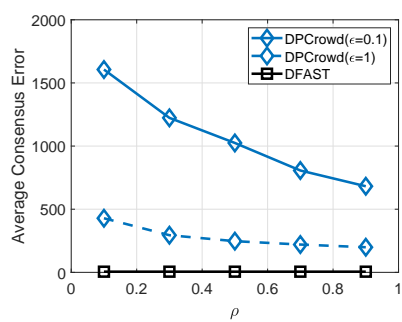

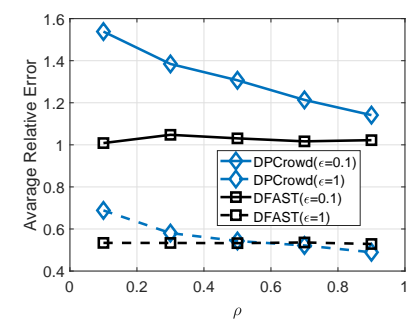

(b) Flu

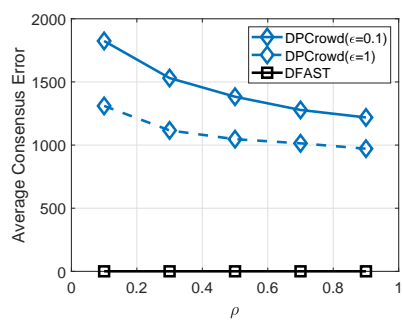

Fig. 5: Average Error vs. Network Density

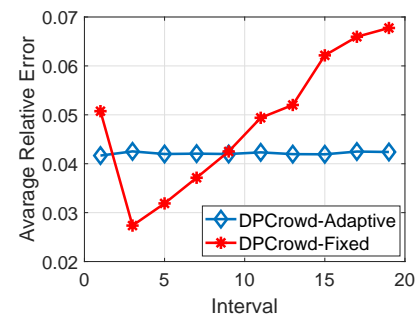

(a) Linear

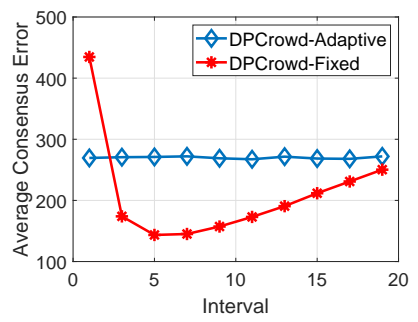

Fig. 6: Average Error vs. Sampling Interval

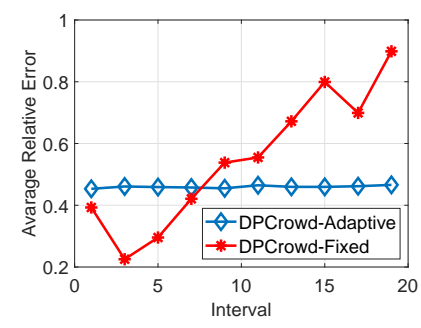

(b) Flu

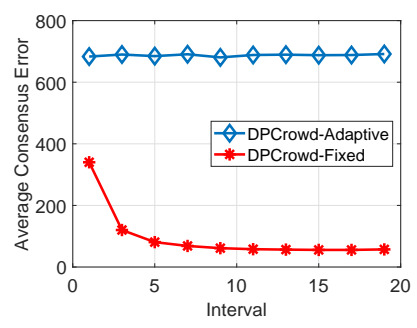

as the synthetic Linear perfectly follows the known process model. Whereas Flu have more fluctuations. Due to wholenetwork broadcast at the expense of large overhead, DFAST can achieve almost the same estimation for all servers and therefore incurs no consensus error. Compared with the noncommunication scheme FAST, DPCrowd has much smaller ACE on both datasets for all privacy levels. The reason is all distributed servers in DPCrowd can exchange and disseminate information iteratively until the convergence. In general, with the increase of $\varepsilon$, ACE for both DPCrowd and FAST drop slowly since fewer noises are added and the differences among servers become smaller.

\section{Estimation Efficiency of DPCrowd}

The communication efficiency of DPCrowd results from two aspects: communication with only one-hop neighbors and communication frequency reduction via sampling based intermittency.

Communication Latency and Overhead: Figs. 8a and $8 \mathrm{~b}$ show the communication latency and overhead of DPCrowd in comparison with DFAST under different network density $\rho$. In Fig. 8a, DPCrowd keeps much less latency since each server only exchanges messages with its one-hop neighbors. Nonetheless, the baseline scheme DFAST incurs much larger latency because the multi-hop broadcast requires much more time to ensure the full dissemination of information. When the network is sparser (smaller $\rho$ ), there are fewer viable routines among servers and cost more communication time. In Fig. 8b, DPCrowd incurs much less communication packets in each sampling timestamp, which increases with $\rho$ slowly. However, the communication overhead of DFAST increases significantly with the density $\rho$. The reason is that the messages have to be broadcast to the whole network via hop by hop. With the 

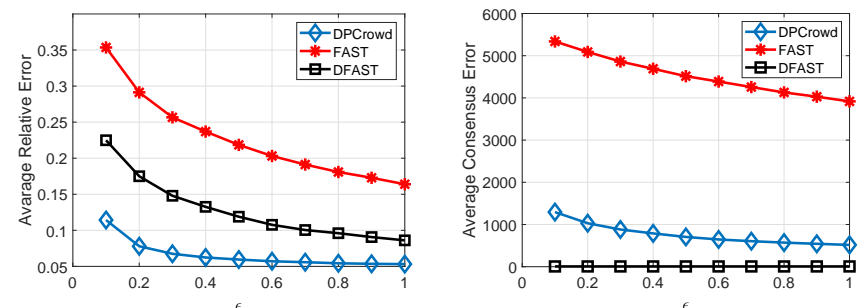

(a) Linear
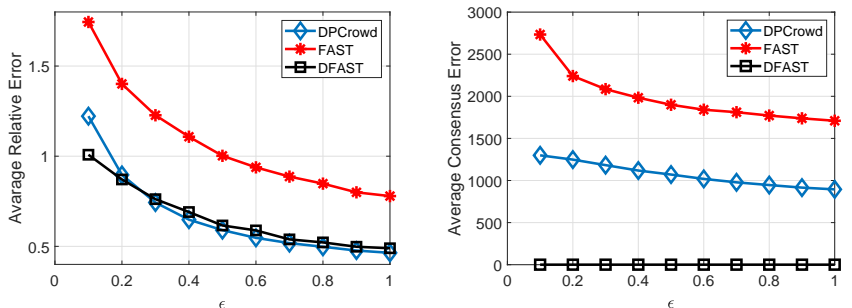

(b) Flu

Fig. 7: Average Error vs. Privacy

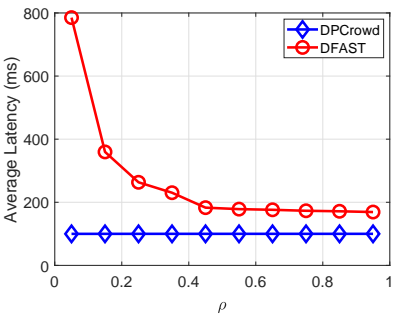

(a) Communication Time

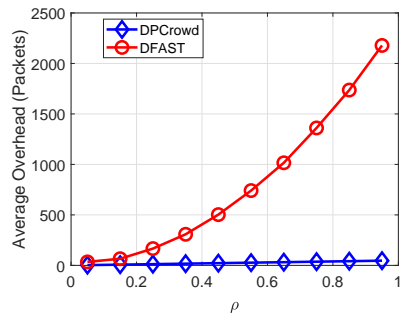

(b) Communication Overhead

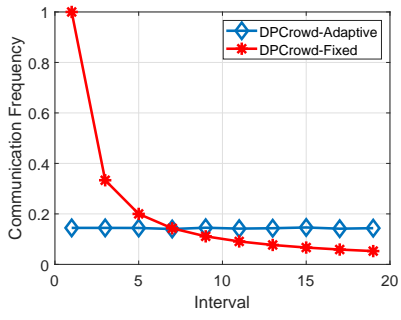

(c) Frequency vs. Interval (Linear)

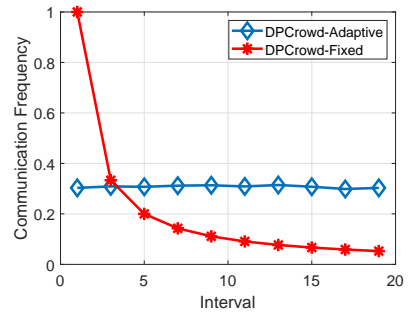

(d) Frequency vs. Interval (Flu)

Fig. 8: Communication Efficiency of DPCrowd

increase of network density, more redundant messages will be forwarded and relayed.

Communication Frequency Reduction: Figs. 8c and 8d depict the average communication frequency of DPCrowd under both the fixed-rate and adaptive rate sampling based intermittent communication strategies. The average communication frequency decreases as the sampling interval increases in the fixed rate strategy, but keeps a lower level for the adaptive sampling strategy given the maximal sampling points ( $0.3 T$ and $0.4 T$ for Linear and Flu, respectively). Together with Fig. 6, we can say the sampling based intermittent communication can effectively reduce the communication frequency and better utilize the privacy budget. Especially, adaptive sampling can better find an optimal sampling interval for DPCrowd with higher efficiency in both communication and privacy preservation.

For conciseness, we mainly compared DPCrowd with DFAST. It should note that, the experimental conclusions of DFAST in terms of communication efficiency also apply to other extension schemes including DBD/DBA, DRescueDP, and DPeGaSus. Apparently, similar reduction in both communication latency and overhead can also be achieved by DPCrowd+ when compared to its counterpart DRescueDP, which is the decentralized extension of RescueDP [9].

\section{Overall Performance of DPCrowd+}

Impact of Windows Size: Fig. 9 shows the estimation utility of DPCrowd+ with the varying windows size $w$, in comparison with other comparable schemes. The AREs of all schemes increase with $w$ for both datasets. This is because given certain privacy budget $\varepsilon$ for a sliding window, larger $w$ means smaller privacy budget for each timestamp and higher perturbation error. The ARE of RescueDP increases with $w$ sharply and reaches the highest in both datasets due to the lack of collaborations among servers. While DPCrowd+ and DPCrowd ${ }_{w}$ show relatively steadily increasing trends. DPCrowd+, compared with DPCrowd ${ }_{w}$, can not only utilize neighbors' knowledge, but also reduce the error via adapting dynamic grouping strategy on the dimensions with small values. Moreover, DPCrowd+ shows superior performance than DPeGaSus and DBD/BA, which is because of further consideration of estimation weights in Eq. (17).

Similarly, DPeGaSus and DBD/BA have almost no consensus error with the cost of whole-network broadcast; and the ACEs of all other schemes increase with $w$ due to less privacy budget allocated on each timestamp. RescueDP shows the largest ACE since there is no collaboration. Although collaborations in DPCrowd $w$ can help to reduce the ACE of RescueDP, higher fluctuations and dimensionality of MultiFlu still lead to high sparsity and make DPCrowd ${ }_{w}$ less effective. In contrast, with the dynamic dimension reduction, DPCrowd+ can achieve better consensus by mitigating the sparsity issue in high-dimensional data.

Tradeoff between Utility and Privacy: Fig. 10 presents the estimation utility of all comparable schemes with respect to different privacy levels $\varepsilon$. For various $\varepsilon$, the ARE of independent estimation scheme RescueDP is the largest due to no communications among distributed servers. Compared with the straightforward extension schemes that incurs great communication latency and overheads, DPCrowd ${ }_{w}$ enforces information exchanges of one-hop neighbors to collaboratively correct the estimation over the network with higher communication efficiency. While DPCrowd ${ }_{w}$ can reduce the estimation error by implementing $w$-event privacy, which, however does not consider the sparsity in multi-dimensional streams. Instead, DPCrowd+ can further reduce the overdose noise on dimensions with small values by adopting the dynamic grouping strategy. Besides, compared with DRescueDP that directly average the whole-network estimations, DPCrowd+ can better fuse the neighboring estimations according to the estimation 

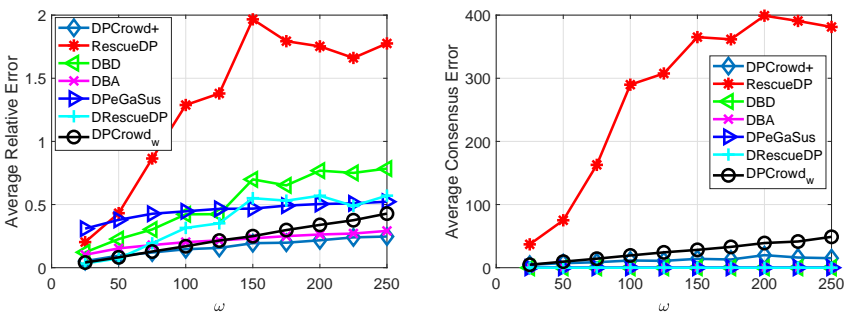

(a) Multi-Linear
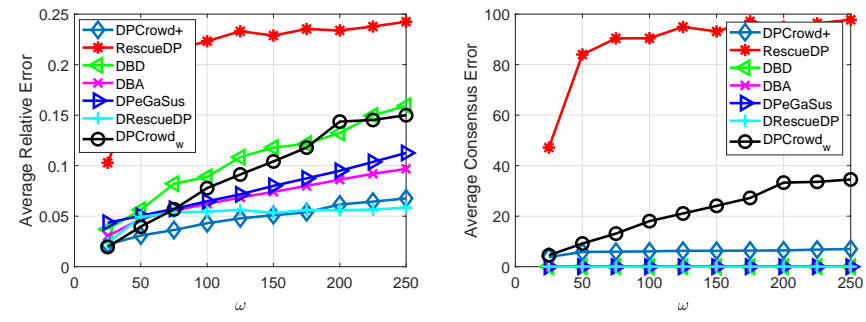

(b) Multi-Flu

Fig. 9: Average Error vs. Window Size

weights (Eq. (17)). Thus, DPCrowd+ shows the smallest ARE among all aforementioned schemes, especially on Multi-Flu. This is because Multi-Flu has more dimensions and is much sparser than Multi-Linear.

In terms of consensus error, DRescueDP, DBD/DBA, and DPeGaSus have nearly no consensus error since expensive all-to-all communications are realized in the whole-network. The ACEs of DPCrowd+, DPCrowd ${ }_{w}$, and the independent estimation scheme RescueDP drops with the increase of $\varepsilon$, which shows that it is easy to achieve consensus when less noise is added. RescueDP has the largest ACE because of no communication among servers. Instead, DPCrowd ${ }_{w}$ shows its superior since message exchange and collaborative correction is leveraged in the estimation. Furthermore, DPCrowd+ has much smaller consensus error as it combines the collaborative correction of in DPCrowd and dynamic grouping to enhance the utility for multi-dimensional data streams.

\section{FINAL REMARKS}

In this paper, we have studied the framework of realtime statistical estimation for multiple distributed servers with crowd-sourced data in a decentralized setting, which enables data sharing and supports IoT-driven smart-world systems. Based on this framework, we first propose a novel scheme with both differential privacy preservation and communication efficiency, DPCrowd, for real-time decentralized statistical estimation on a finite crowd-sourced data stream. In specific, DPCrowd on distributed servers can achieve a consensus estimate of the true statistics by identifying the temporal correlations in data streams and exchanging the perturbed information intermittently with only one-hop neighbors. Additionally, as an extension for practical decentralized statistical estimation on infinite high-dimensional crowd-sourced data streams, we further propose DPCrowd+ to realize not only $w$-event DP, but also dimensional reduction by learning the sparse structure of multi-dimensional data. Extensive experimental results on realworld datasets show that our proposed schemes DPCrowd and DPCrowd+ are efficient and effective in obtaining accurate and consensus real-time statistical estimation for distributed servers on crowd-sourced data streams while guaranteeing sufficient DP for crowd-sourcing users.

\section{REFERENCES}

[1] X. Zheng and Z. Cai, "Privacy-preserved data sharing towards multiple parties in industrial iots," IEEE J. Sel. Areas Commun., vol. PP, pp. $1-12,2020$.
[2] X. Liu, C. Qian, W. G. Hatcher, H. Xu, W. Liao, and W. Yu, "Secure internet of things (iot)-based smart-world critical infrastructures: Survey, case study and research opportunities," IEEE Access, vol. 7, pp. $79523-$ 79544, 2019.

[3] N. Kumar, S. Zeadally, and J. J. P. C. Rodrigues, "Vehicular delaytolerant networks for smart grid data management using mobile edge computing," IEEE Commun. Mag., vol. 54, no. 10, pp. 60-66, 2016.

[4] J. Lin, W. Yu, N. Zhang, X. Yang, H. Zhang, and W. Zhao, "A survey on internet of things: Architecture, enabling technologies, security and privacy, and applications," IEEE Internet Things J., vol. 4, no. 5, pp. 1125-1142, 2017.

[5] P. Huang, L. Guo, M. Li, and Y. Fang, "Practical privacy-preserving ecgbased authentication for iot-based healthcare," IEEE Internet Things J., vol. 6, no. 5, pp. 9200-9210, 2019.

[6] H. Wang, M. S. M. H. Fang, and C. Wang, Wireless Health, 2016.

[7] M. Braverman, A. Garg, T. Ma, H. L. Nguyen, and D. P. Woodruff, "Communication lower bounds for statistical estimation problems via a distributed data processing inequality," in Proc. ACM STOC, 2016, pp. 1011-1020.

[8] M. I. Jordan, J. D. Lee, and Y. Yang, "Communication-efficient distributed statistical inference," J. Amer. Statist. Assoc., vol. 114, no. 526, pp. 668-681, 2019.

[9] Q. Wang, Y. Zhang, X. Lu, Z. Wang, Z. Qin, and K. Ren, "Rescuedp: Real-time spatio-temporal crowd-sourced data publishing with differential privacy," in Proc. IEEE INFOCOM, 2016, pp. 1-9.

[10] Z. Wang, X. Pang, Y. Chen, H. Shao, Q. Wang, L. Wu, H. Chen, and H. Qi, "Privacy-preserving crowd-sourced statistical data publishing with an untrusted server," IEEE Trans. Mobile Comput., vol. 18, no. 6, pp. 1356-1367, 2018.

[11] A. Zanella, N. Bui, A. Castellani, L. Vangelista, and M. Zorzi, "Internet of things for smart cities," IEEE Internet Things J., vol. 1, no. 1, pp. 22-32, 2014.

[12] X. Li, S. Liu, F. Wu, S. Kumari, and J. J. Rodrigues, "Privacy preserving data aggregation scheme for mobile edge computing assisted iot applications," IEEE Internet Things J., vol. 6, no. 3, pp. 4755-4763, 2018.

[13] N. Sonehara, I. Echizen, and S. Wohlgemuth, "Isolation in cloud computing and privacy-enhancing technologies," Business \& information systems engineering, vol. 3, no. 3, p. 155, 2011.

[14] Z. Zhang, S. He, J. Chen, and J. Zhang, "Reap: An efficient incentive mechanism for reconciling aggregation accuracy and individual privacy in crowdsensing," IEEE Trans. Inf. Forensics Security, vol. 13, no. 12, pp. 2995-3007, 2018.

[15] A. Asadi, Q. Wang, and V. Mancuso, "A survey on device-to-device communication in cellular networks," IEEE Commun. Surveys Tuts., vol. 16, no. 4, pp. 1801-1819, 2014.

[16] S. S. Stankovic, M. S. Stankovic, and D. M. Stipanovic, "Decentralized parameter estimation by consensus based stochastic approximation," IEEE Trans. Autom. Control, vol. 56, no. 3, pp. 531-543, 2010.

[17] S. Kar, J. M. Moura, and K. Ramanan, "Distributed parameter estimation in sensor networks: Nonlinear observation models and imperfect communication," IEEE Trans. Inf. Theory, vol. 58, no. 6, pp. 3575-3605, 2012.

[18] C. Dwork and A. Roth, "The algorithmic foundations of differential privacy," Foundations \& Trends $₫$ in Theoretical Computer Science, vol. 9, no. 3, 2013.

[19] M. U. Hassan, M. H. Rehmani, and J. Chen, "Differential privacy techniques for cyber physical systems: a survey," IEEE Commun. Surveys Tuts, 2019.

[20] S. Su, P. Tang, X. Cheng, R. Chen, and Z. Wu, "Differentially private 


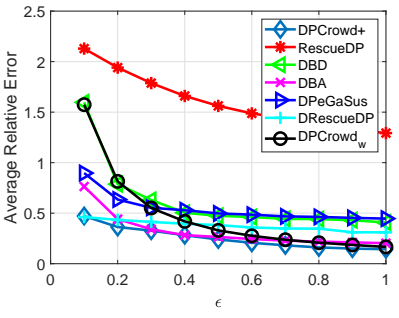

(a) Multi-Linear

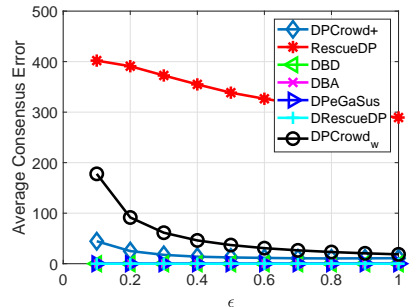

Fig. 10: Average Error vs. Privacy multi-party high-dimensional data publishing," in Proc. IEEE ICDE, 2016, pp. 205-216.

[21] X. Yang, T. Wang, X. Ren, and W. Yu, "Survey on improving data utility in differentially private sequential data publishing," IEEE Trans. Big Data, pp. 1-17, 2017.

[22] X. Ren, C.-M. Yu, W. Yu, S. Yang, X. Yang, J. A. McCann, and S. Y. Philip, "Lopub: High-dimensional crowdsourced data publication with local differential privacy," IEEE Trans. Inf. Forensics Security, vol. 13, no. 9, pp. 2151-2166, 2018.

[23] T. Wang, X. Yang, X. Ren, W. Yu, and S. Yang, "Locally private high-dimensional crowdsourced data release based on copula functions," IEEE Trans. Services Comput., pp. 1-16, 2019.

[24] L. Fan and L. Xiong, "An adaptive approach to real-time aggregate monitoring with differential privacy," IEEE Trans. Knowl. Data Eng, vol. 26, no. 9, pp. 2094-2106, 2014.

[25] T. Wang, J. Zhao, H. Yu, J. Liu, X. Yang, X. Ren, and S. Shi, "Privacypreserving crowd-guided AI decision-making in ethical dilemmas," in Proc. ACM CIKM, 2019, pp. 1311-1320.

[26] Z. Huang, S. Mitra, and G. Dullerud, "Differentially private iterative synchronous consensus," in Proc. ACM WPES, 2012, pp. 81-90.

[27] Z. Huang, S. Mitra, and N. Vaidya, "Differentially private distributed optimization," in Proc. ACM ICDCN, 2015, pp. 1-10.

[28] C. Li, P. Zhou, L. Xiong, Q. Wang, and T. Wang, "Differentially private distributed online learning," IEEE Trans. Knowl. Data Eng., vol. 30, no. 8, pp. 1440-1453, 2018.

[29] F. D. McSherry, "Privacy integrated queries: an extensible platform for privacy-preserving data analysis," in Proc. ACM SIGMOD, 2009, pp. 19-30.

[30] C. Dwork, M. Naor, T. Pitassi, and G. N. Rothblum, "Differential privacy under continual observation," in Proc. ACM STOC, 2010, pp. 715-724.

[31] C. Dwork, "Differential privacy," in Proc. ICALP, 2006, pp. 1-12.

[32] — "Differential privacy in new settings," in Proc. ACM-SIAM SODA, 2010, pp. 174-183.

[33] D. Mir, S. Muthukrishnan, A. Nikolov, and R. N. Wright, "Pan-private algorithms via statistics on sketches," in Proc. ACM PODS, 2011, pp. $37-48$.

[34] T.-H. H. Chan, M. Li, E. Shi, and W. Xu, "Differentially private continual monitoring of heavy hitters from distributed streams," in Proc. PETS. Springer, 2012, pp. 140-159.

[35] Y. Chen, A. Machanavajjhala, M. Hay, and G. Miklau, "Pegasus: Dataadaptive differentially private stream processing," in Proc. ACM CCS, 2017, pp. 1375-1388.

[36] G. Kellaris, S. Papadopoulos, X. Xiao, and D. Papadias, "Differentially private event sequences over infinite streams," Proceedings of the VLDB Endowment, vol. 7, no. 12, pp. 1155-1166, 2014.

[37] Q. Wang, Y. Zhang, X. Lu, Z. Wang, Z. Qin, and K. Ren, "Real-time and spatio-temporal crowd-sourced social network data publishing with differential privacy," IEEE Trans. Dependable Secure Comput., vol. 15, no. 4, pp. 591-606, 2016.

[38] G. Ács and C. Castelluccia, "I have a dream!(differentially private smart metering)," in International Workshop on Information Hiding. Springer, 2011, pp. 118-132.

[39] S. Goryczka and L. Xiong, "A comprehensive comparison of multiparty secure additions with differential privacy," IEEE Trans. Dependable Secure Comput., vol. 14, no. 5, pp. 463-477, 2017.

[40] D. Alhadidi, N. Mohammed, B. C. Fung, and M. Debbabi, "Secure distributed framework for achieving $\varepsilon$-differential privacy," in Proc. PETS, 2012, pp. 120-139.

[41] Y. Hong, J. Vaidya, H. Lu, P. Karras, and S. Goel, "Collaborative search log sanitization: Toward differential privacy and boosted utility," IEEE Trans. Dependable Secure Comput., vol. 12, no. 5, pp. 504-518, 2015.
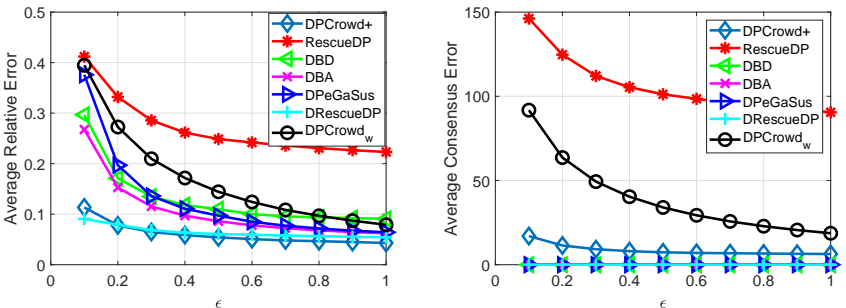

(b) Multi-Flu

[42] Ú. Erlingsson, V. Pihur, and A. Korolova, "Rappor: Randomized aggregatable privacy-preserving ordinal response," in Proc. ACM CCS, 2014, pp. $1054-1067$

[43] E. V. Belmega, L. Sankar, and H. V. Poor, "Enabling data exchange in two-agent interactive systems under privacy constraints," IEEE J. Sel. Topics Signal Process., vol. 9, no. 7, pp. 1285-1297, 2015.

[44] S. Truex, N. Baracaldo, A. Anwar, T. Steinke, H. Ludwig, R. Zhang, and Y. Zhou, "A hybrid approach to privacy-preserving federated learning," in Proc. ACM AISec@CCS, 2019, pp. 1-11.

[45] L. Zhao, Q. Wang, Q. Zou, Y. Zhang, and Y. Chen, "Privacy-preserving collaborative deep learning with unreliable participants," IEEE Trans. Inf. Forensics Security, vol. 15, pp. 1486-1500, 2019.

[46] R. C. Geyer, T. Klein, and M. Nabi, "Differentially private federated learning: A client level perspective," arXiv preprint arXiv:1712.07557, 2017.

[47] T. Zhang and Q. Zhu, "Dynamic differential privacy for admm-based distributed classification learning," IEEE Trans. Inf. Forensics Security, vol. 12, no. 1, pp. 172-187, 2017.

[48] Z. Wang, M. Song, Z. Zhang, Y. Song, Q. Wang, and H. Qi, "Beyond inferring class representatives: User-level privacy leakage from federated learning," in Proc. IEEE INFOCOM. IEEE, 2019, pp. 2512-2520.

[49] N. Li, M. Lyu, D. Su, and W. Yang, "Differential privacy: From theory to practice," Synthesis Lectures on Information Security, Privacy, \& Trust, vol. 8, no. 4, pp. 1-138, 2016.

[50] Y. Cao, M. Yoshikawa, Y. Xiao, and L. Xiong, "Quantifying differential privacy in continuous data release under temporal correlations," IEEE Trans. Knowl. Data Eng., vol. 31, no. 7, pp. 1281-1295, 2018.

[51] F. McSherry, "Differential privacy and correlated data," https://github.com/frankmcsherry/blog/blob/master/posts/2016-08-29.md.

[52] S. Song, Y. Wang, and K. Chaudhuri, "Pufferfish privacy mechanisms for correlated data," in Proc. ACM CIKM, 2017, pp. 1291-1306.

[53] J. Kang, Z. Xiong, D. Niyato, S. Xie, and J. Zhang, "Incentive mechanism for reliable federated learning: A joint optimization approach to combining reputation and contract theory," IEEE Internet Things J., vol. 6, no. 6, pp. 10700-10 714, 2019.

[54] R. Olfati-Saber, "Kalman-consensus filter: Optimality, stability, and performance," in Proc. IEEE CDC, 2009, pp. 7036-7042.

[55] L. Fan, L. Bonomi, L. Xiong, and V. Sunderam, "Monitoring web browsing behavior with differential privacy," in Proc. ACM WWW, 2014, pp. 177-188. 